\title{
The one-phase Hele-Shaw Problem with singularities.
}

\author{
David Jerison and Inwon Kim \\ Department of Mathematics, MIT
}

May 25, 2005

\begin{abstract}
In this paper we analyze viscosity solutions of the one phase HeleShaw problem in the plane and the corresponding free boundaries near a singularity. We find, up to order of magnitude, the speed at which the free boundary moves starting from a wedge, cusp, or finger-type singularity. Maximum principle-type arguments play a key role in the analysis.
\end{abstract}

\section{Introduction}

Consider a compact set $K \subset \mathbb{R}^{2}$ with smooth boundary $\partial K$. Suppose that a bounded domain $\Omega$ contains $K$ and let $\Omega_{0}=\Omega-K$ and $\Gamma_{0}=\partial \Omega$ (Figure 1 ). Note that $\partial \Omega_{0}=\Gamma_{0} \cup \partial K$.

Let $u_{0}$ be a harmonic function in $\Omega_{0}$ with boundary data 1 on $\partial K$ and zero

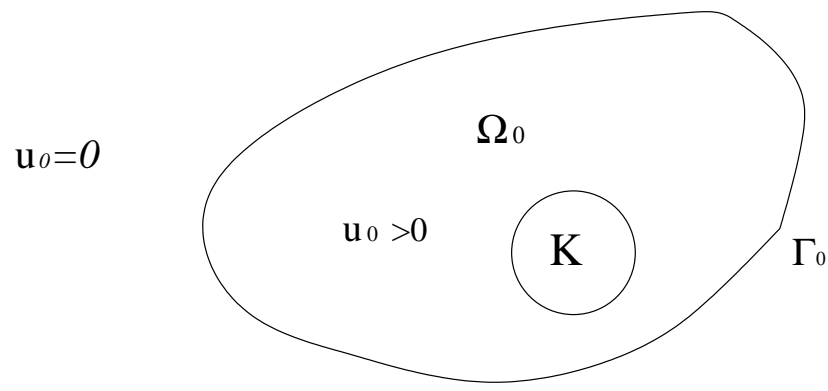

Figure 1. 
on $\Gamma_{0}$. Let $u(x, t)$ solve the one-phase Hele-Shaw problem:

$$
\begin{cases}-\Delta u=0 & \text { in }\{u>0\} \cap Q, \\ u_{t}-|\nabla u|^{2}=0 & \text { on } \partial\{u>0\} \cap Q, \\ u(x, 0)=u_{0}(x) ; & u(x, t)=1 \text { for } x \in \partial K .\end{cases}
$$

where $Q=\left(\mathbb{R}^{2}-K\right) \times(0, \infty)$.

We refer to $\Gamma_{t}(u):=\partial\{u(\cdot, t)>0\}-\partial K$ as the free boundary of $u$ at time $t$. Note that if $u$ is smooth up to the free boundary, then the free boundary moves with normal velocity $V=u_{t} /|\nabla u|$, and hence the second equation in (HS) implies that $V=|\nabla u|$. The classical Hele-Shaw problem models an incompressible viscous fluid which occupies part of the space between two parallel, nearby plates.

Our goal is to investigate the free boundary behavior near $t=0$ when the initial free boundary $\Gamma_{0}$ contains a singularity at a point. To formulate the sense in which singular solutions solve the Hele-Shaw equation, we use a notion of viscosity solutions whose existence and uniqueness were proved in [K1]. For rigorous statements, see Section 2.

For simplicity, consider three model cases. Assume that $\Gamma_{0}$ is $C^{1,1}$ except at the origin. Denote the ball (disk) in $\mathbb{R}^{2}$ of radius $r$ with center $P$ by $B_{r}(P)$.

Case $A$. Wedge with angle $\theta_{0}$ (Figure 2-A): $K=\bar{B}_{1}\left(P_{0}\right), P_{0}=(0,10)$ and

$$
\Omega \cap B_{3}(0)=\left\{(x, y): y \leq|x| \cot \frac{\theta_{0}}{2}\right\} \cap B_{3}(0) .
$$

Case B. Cusp (Figure 2-B): $K=\bar{B}_{1}\left(P_{0}\right), P_{0}=(0,5)$ and

$$
\Omega \cap B_{3}(0)=\left\{(x, y): y \leq|x|^{1 / 2}\right\} \cap B_{3}(0) .
$$

Case $C$. Two fingers touching (Figure 2-C):

$$
K=\bar{B}_{1 / 10}((-1,0)) \cup \bar{B}_{1 / 10}((1,0)) ; \quad \Omega=B_{1}((-1,0)) \cup B_{1}((1,0)) .
$$

It is natural to ask if singularities of these types can occur at a positive time. Obviously, Case $\mathrm{C}$ occurs at a positive time when two radially symmetric solutions with disjoint support collide, and, more generally, a singularity of this type occurs when two expanding fingers meet. Cusps (Case B) and angles (Case A) with $0<\theta_{0}<\pi$ also occur at positive times, as demonstrated in $[\mathrm{H}]$ and [KLV].

Consider a point $P \in B_{1}(0) \cap\left(\mathbb{R}^{2}-\bar{\Omega}\right)$, and define

$$
t(P)=\sup \{t>0: u(P, t)=0\} .
$$

In other words $t(P)$ is the time the free boundary reaches $P$. Our main result is an estimate on the size of $t(P)$ in terms of the initial data. Define $\delta=$ $\delta(P)=\operatorname{dist}(P, \bar{\Omega})$. Choose any point $z=\zeta(P)$ in $\Omega$ such that $|P-z|=2 \delta$ and $\operatorname{dist}(z, \partial \Omega) \geq \delta / 2$. 


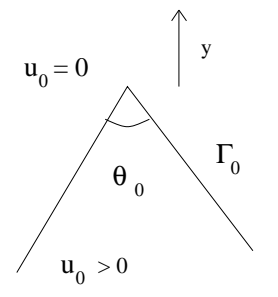

$2-\mathrm{A}$

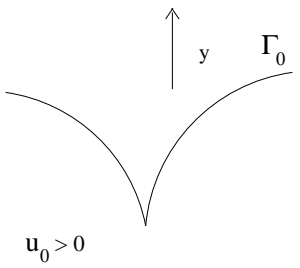

2-B

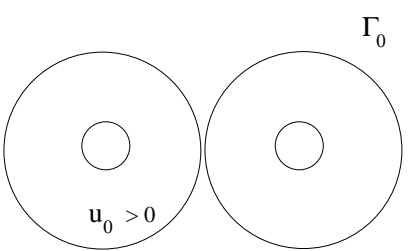

$2-\mathrm{C}$

Figure 2.

Theorem 1.1. (Main Theorem) Let $u$ be a viscosity solution to the HeleShaw equation given by Theorem 2.8. In Case $A$, provided $\theta_{0}>\pi / 2$ and in Cases $B$ and $C$,

$$
t(P) \simeq \delta(P)^{2} / u_{0}(\zeta(P))
$$

where $a \simeq b$ means that $a / b$ is bounded above and below by positive constants.

In Case $\mathrm{A}$, the constant in (1.2) is uniform as the angle $\theta_{0}$ tends to $2 \pi$ (Lemma 4.1). Because of this uniformity, it is natural to expect the same estimate holds for the cusp (Case B). The fact that it also holds for the fingers (Case $\mathrm{C}$ ) is interesting because of the change in connectivity of the region.

The meaning of (1.2) is more transparent when it is expressed in terms of the average speed of the free boundary, $\delta(P) / t(P)$. According to (1.2),

$$
\delta(P) / t(P) \simeq \delta(P) u_{0}(\zeta(P)) / \delta(P)^{2}=u_{0}(\zeta(P)) / \delta(P) .
$$

One should interpret $u_{0}(\zeta(P)) / \delta(P)$ as an approximate slope of the graph of $u_{0}$. Well-known estimates for harmonic functions show that $u_{0}(z) / \delta$ is comparable to the average of $\left|\nabla u_{0}\right|$ on any arc of $\Gamma_{0}$ of size $\delta$ near $z$ or $P$. Recall that (for smooth solutions) the normal velocity of the free boundary is given by $V=|\nabla u|$. So it is natural to expect that the average velocity $\delta(P) / t(P)$ is an average of $|\nabla u|$. (1.2) says that the average speed of the free boundary near $P$ over time interval of length $t(P)$ is comparable to the average of the initial velocity $\left|\nabla u_{0}\right|$ on any arc of $\Gamma_{0}$ of length $\delta(P)$ near $P$.

(1.2) is not valid for acute angles $\theta_{0}<\pi / 2$. In fact, $\delta(P)^{2} / u_{0}(\zeta(P))$ tends to infinity as $P$ tends to the vertex. This reflects a qualitative change in the speed of free boundary. We know from $[\mathrm{KLV}]$ that the vertex does begin to move eventually, but only after a positive "waiting time." In other words $t(P)$ tends to a positive (but not infinite) number as $P$ tends to the vertex. Therefore, (1.2) would have to be modified to cover the case of acute angles.

Explicit bounds and further results. We now formulate what (1.2) says explicitly. This can be done because in our cases the order of the magnitude of $u_{0}$ is explicit. 


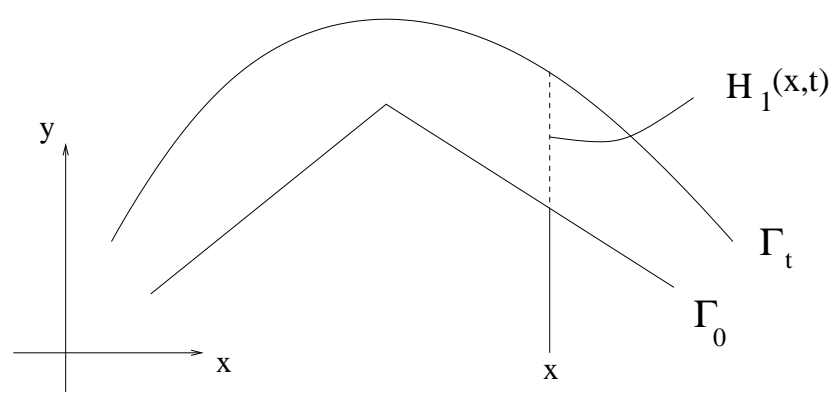

Figure 3.

Theorem 1.2. Let $u$ be a viscosity solution of (HS) given by Theorem 2.4.

a) In case (A), with $\pi / 2<\theta_{0}<2 \pi$ and $\alpha=2-\pi / \theta_{0}$,

$$
t(P) \simeq \delta(P)|P|^{\alpha-1}
$$

b) In case (B), $t(P) \simeq \delta(P)|P|^{1 / 2}$.

c) In case $(C), t(P) \simeq \delta(P)$.

These estimates give the order of magnitude of the normal and the vertical displacement of the free boundary, uniformly in a neighborhood of the origin in space-time. Define the vertical displacement (figure 3) of the free boundary by $H_{1}(x, t)=y(x, t)-y(x, 0)$, where

$$
y(x, t)=\sup \left\{y:(x, y) \in B_{1}(0) \cap \Gamma_{t}(u)\right\}
$$

Then Theorem 1.2 can be equivalently stated in terms of $H_{1}(x, t)$ as below:

Corollary 1.3. Let $u$ be a viscosity solution of (HS) given by Theorem 2.4. For $(x, t)$ small, the vertical displacement $H_{1}$ has the following order of magnitude.

a) In case (A),

$$
\begin{gathered}
H_{1}(x, t) \simeq \max \left[t^{1 / \alpha},|x|^{1-\alpha} t\right], \quad \text { for } \pi / 2<\theta_{0} \leq \pi ; \\
H_{1}(x, t) \simeq \min \left[t^{1 / \alpha},|x|^{1-\alpha} t\right], \quad \text { for } \pi \leq \theta_{0}<2 \pi .
\end{gathered}
$$

b) In case $(B), H_{1}(x, t) \simeq \min \left(t^{2 / 5},|x|^{-3 / 4} t\right)$

c) In case $(C), H_{1}(x, t) \simeq \min \left(t^{1 / 2},|x|^{-1 / 2} t\right)$

In the proof of Theorem 1.2 in section $3-5$ we will prove the equivalent statements in Coroally 1.3.

Finally, we prove smoothness of the free boundary for small positive times.

Theorem 1.4. In Case $A$, for $\pi / 2<\theta_{0}<2 \pi$,

(a) $H_{1}(0, t) t^{-1 / \alpha}$ converges to a positive limit as $t \rightarrow 0$. 
(b) Moreover, the free boundary remains Lipschitz in $B_{1}$ for a short time. If, in addition, the initial domain is star-shaped, then the free boundary is smooth (even real analytic) in space for small positive time and the free boundary condition is satisfied in the classical sense.

Remarks. We expect that smoothness in the space variable in Case A, $\pi / 2<\theta_{0}<2 \pi$, is valid without the additional hypothesis that the domain is star-shaped. Smoothness in the time variable cannot be expected without some kind of global hypothesis on the initial data. We also expect corresponding smoothness results for Case B and C.

Lemma 4.1 and the other arguments in section 4 can be applied to prove that (1.2) holds with any convex cusp-type singularity in place of the explicit $1 / 2$-order cusp given in Case B.

The method of barrier functions developed here can be applied to singularities in higher dimensions with rotational symmetry. For example see Theorem 3.10 .

In all cases, obtaining the lower bound for the speed of the free boundary (i.e., the upper bound on $t(P)$ ) is relatively easy. It is proved in Proposition 3.6 using a radial subsolution. On the other hand, to obtain the upper bound for the speed of the free boundary requires more subtle choice of barrier functions that takes into account the global behavior of the free boundary.

Outline of the paper. In Section 1 we set up notations. In Section 2 we introduce the notion of viscosity solutions. In Section 3, we analyze Case A, the wedge. We construct explicit barrier functions based on maximal principle-type arguments and properties of harmonic functions in Lipschitz domains. The asymptotics mentioned in Theorem 1.4 (a) above depend on the blow-up solutions constructed in $[\mathrm{KLV}]$. To obtain the (spatial) Lipschitz regularity for small time, we use a reflection argument. The higher regularity proved here depends on regularity results of [K2]-[K3]. In Section 4 we investigate Case B, the cusp singularity. We approximate the cusp shape with angles and use estimates obtained in Case A. The main point is uniformity of bounds as the angle $\theta_{0}$ increases to $2 \pi$ (Lemma 4.1). Note that for the cusp case the blow-up limit of $\Gamma_{0}$ at the origin is a vertical slit and the vertical slit disappears instantly under Hele-Shaw flow. Hence the limiting case does not give sufficient information on how fast the free boundary moves. Finally in Section 5 we discuss Case C, the two-finger singularity. The arguments are similar to Section 4.

Throughout the paper we denote $\beta_{0}=\pi-\theta_{0} / 2, \beta=\tan \beta_{0}$ and $\alpha=2-\pi / \theta_{0}$ for given wedge angle $\theta_{0} \in(\pi / 2,2 \pi)$.

\section{$2 \quad$ Viscosity solutions}

Consider a domain $D \subset \mathbb{R}^{n}$ and an interval $I \subset \mathbb{R}$. For a nonnegative real valued function $u(x, t)$ defined for $(x, t) \in D \times I$, define 


$$
\begin{aligned}
& \Omega(u)=\{(x, t) \in D \times I: u(x, t)>0\}, \quad \Omega_{t}(u)=\{x \in D: u(x, t)>0\} \\
& \Gamma(u)=\partial \Omega(u)-\partial(D \times I), \quad \Gamma_{t}(u)=\partial \Omega_{t}(u)-\partial D .
\end{aligned}
$$

Let us recall the notion of viscosity solutions of (HS) defined in [K1]. Roughly speaking, viscosity sub and supersolutions are defined by comparison with local (smooth) super and subsolutions. In particular classical solutions of (HS) are also viscosity sub and supersolutions of (HS). Let $Q=\left(\mathbb{R}^{n}-K\right) \times(0, \infty)$ and let $\Sigma$ be a cylindrical domain $D \times(a, b) \subset \mathbb{R}^{n} \times \mathbb{R}$, where $D$ is an open subset of $\mathbb{R}^{n}$.

Definition 2.1. A nonnegative upper semicontinuous function $u$ defined in $\Sigma$ is a viscosity subsolution of $(H S)$ if

(a) for each $a<T<b$ the set $\overline{\Omega(u)} \cap\{t \leq T\}$ is bounded; and

(b) for every $\phi \in C^{2,1}(\Sigma)$ such that $u-\phi$ has a local maximum in $\overline{\Omega(u)} \cap\{t \leq$ $\left.t_{0}\right\} \cap \Sigma$ at $\left(x_{0}, t_{0}\right)$,

(i) $-\Delta \phi\left(x_{0}, t_{0}\right) \leq 0 \quad$ if $u\left(x_{0}, t_{0}\right)>0$.

(ii) $\quad\left(\phi_{t}-|\nabla \phi|^{2}\right)\left(x_{0}, t_{0}\right) \leq 0 \quad$ if $\left(x_{0}, t_{0}\right) \in \Gamma(u)$ and if $-\Delta \phi\left(x_{0}, t_{0}\right)>0$.

Note that because $u$ is only upper lowercontinuous there may be points of $\Gamma(u)$ at which $u$ is positive.

Definition 2.2. A nonnegative lower semicontinuous function $v$ defined in $\Sigma$ is a viscosity supersolution of $(H S)$ if for every $\phi \in C^{2,1}(\Sigma)$ such that $v-\phi$ has a local minimum in $\Sigma \cap\left\{t \leq t_{0}\right\}$ at $\left(x_{0}, t_{0}\right)$,

$$
\begin{gathered}
\text { (i) } \quad-\Delta \phi\left(x_{0}, t_{0}\right) \geq 0 \quad \text { if } v\left(x_{0}, t_{0}\right)>0, \\
\text { (ii) } \quad \text { If }\left(z_{0}, t_{0}\right) \in \Gamma(v),|\nabla \phi|\left(x_{0}, t_{0}\right) \neq 0 \text { and } \\
-\Delta \varphi\left(x_{0}, t_{0}\right)<0,
\end{gathered}
$$

then

$$
\left(\phi_{t}-|\nabla \phi|^{2}\right)\left(x_{0}, t_{0}\right) \geq 0 .
$$

Definition 2.3. $u$ is a viscosity subsolution of (HS) with initial data $u_{0}$ and fixed boundary data $f>0$ if

(a) $u$ is a viscosity subsolution in $\bar{Q}$,

(b) $u=u_{0}$ at $t=0 ; u \leq f$ on $\partial K$;

(c) $\overline{\Omega(u)} \cap\{t=0\}=\overline{\Omega\left(u_{0}\right)}$; 
Definition 2.4. We say that a subsolution in $D \times(0, b)$ has initial data $u_{0}$ if $u=u_{0}$ at $t=0$ and $\overline{\Omega(u)} \cap\{t=0\}=\overline{\Omega\left(u_{0}\right)}$. We say that a supersolution in $D \times(0, b)$ has initial data $u_{0}$ if $u=u_{0}$ at $t=0$.

For a nonnegative real valued function $u(x, t)$ defined in a cylindrical domain $D \times(a, b)$,

$$
u^{*}(x, t)=\limsup _{(\zeta, \tau) \in D \times I \rightarrow(x, t)} u(\zeta, \tau) .
$$

Note that the limsup permits times in the future of $t, s>t$.

Definition 2.5. $u$ is a viscosity solution of (HS) (with boundary data $u_{0}$ and $f$ ) if $u$ is a viscosity supersolution and $u^{*}$ is a viscosity subsolution of (HS) (with boundary data $u_{0}$ and $f$.)

Definition 2.6. We say that a pair of functions $u_{0}, v_{0}: \bar{D} \rightarrow[0, \infty)$ are (strictly) separated (denoted by $u_{0} \prec v_{0}$ ) in $D \subset \mathbb{R}^{n}$ if

(i) the support of $u_{0}, \operatorname{supp}\left(u_{0}\right)=\overline{\left\{u_{0}>0\right\}}$ restricted in $\bar{D}$ is compact and

(ii) in $\operatorname{supp}\left(u_{0}\right) \cap \bar{D}$ the functions are strictly ordered:

$$
u_{0}(x)<v_{0}(x)
$$

(Typically $u_{0}$ is upper semicontinuous and $v_{0}$ is lower semicontinuous. In this case, $\operatorname{supp}\left(u_{0}\right) \subset \operatorname{int}\left(\operatorname{supp}\left(v_{0}\right)\right)$ since $\left\{v_{0}>0\right\}$ is open.)

The following two theorems state important properties of viscosity solutions.

Theorem 2.7. (Comparison Principle, [K1]) (a) Let $u$ and $v$ be viscosity sub and supersolutions, respectively, of $(H S)$ in $Q=\left(\mathbb{R}^{n}-K\right) \times(0, \infty)$ with initial data $u_{0}$ and $v_{0}$. If $u_{0} \prec v_{0}$ and $u<v$ for $z \in \partial K$, then $u(\cdot, t) \prec v(\cdot, t)$ in $\mathbb{R}^{2}-K$ for $t>0$.

(b)(localized version) Let $u, v$ be respectively viscosity sub-and supersolutions in $D \times(0, T) \subset Q$ with initial data $u_{0} \prec v_{0}$. If $u \leq v$ on $\partial D$ and $u<v$ on $\partial D \cap \overline{\{u>0\}}$ for $0 \leq t<T$, then $u(\cdot, t) \prec v(\cdot, t)$ in $\bar{D}$ for $t \in[0, T)$.

(c) If $u_{0} \leq v_{0}, u \leq v$ for $z \in \partial K$ and $\Gamma(v)$ moves immediately at $t=0$, then for any $\epsilon>0$

$$
u(\cdot, t) \prec(1+\epsilon) v(\cdot, t+\epsilon) \text { for } t \geq 0 .
$$

Let $\Omega \subset \mathbb{R}^{2}$ be a bounded open set whose boundary consists of two disjoint compact sets $K_{1}$ and $K_{2}$. For $n=2$ we assume that $K_{i}$ has finite number of connected components and that no such component is an isolated point. Define

$$
u=H\left(a, K_{1}, \Omega\right)
$$

to be the continuous harmonic function in $\Omega$ with boundary data $u=a$ on $K_{1}$ and $u=0$ on $K_{2}=(\partial \Omega) \backslash K_{1}$. (Such a function $u$ exists under the assumptions on $\Omega$ for $n=2$. For $n>2$ we can, for example, assume that $\Omega$ is a Lipschitz domain.) 
Theorem 2.8. Let $K$ be a smoothly bounded compact set. Let $\Omega$ be an open, bounded, connected set in $\mathbb{R}^{n}$ such that $\partial \Omega=K_{1} \cup K_{2}$ as above with $K_{1}=\partial K$. Let $u_{0}=H(1, \partial K, \Omega)$. Then the infimum of viscosity supersolutions defined on $Q=\left(\mathbb{R}^{n} \backslash K\right) \times[0, \infty)$ with initial data $u_{0}$ and boundary data $u(z, t) \geq$ 1 for $z \in \partial K$ is a viscosity solution with initial data $u_{0}$ and boundary data $u=1$ on $\partial K$. If $\Gamma(u)$ immediately moves at $t=0$ (in other words, $\Gamma_{0}(u) \subset$ int $\Omega_{t}(u)$ for $\left.t>0\right)$, then $u$ is the unique viscosity solution of $(H S)$ with its boundary data. Furthermore, $u$ is increasing in $t$ and $u(\cdot, t)$ is harmonic in $\Omega_{t}(u)$.

Theorems 2.7 and 2.8 are proved for the most part in [K1]. The further property that $u$ is increasing in $t$ follows from the definition of a viscosity solution. The fact that $u$ is harmonic in $z$ for each $t$ and achieves it boundary values on $\partial K$ is proved in [K2].

Theorem 2.7 is proved in [K1]. Theorem 2.8 with $C^{1,1}$ hypersurface $\Gamma_{0}$ is proved in [K1]. This result and standard arguments using Perron's method yield Theorem 2.8. The property that $u$ is increasing in $t$ follows from the definition of a viscosity solution. The fact that $u$ is harmonic in $z$ for each $t$ and achieves its boundary values on $\partial K$ is proved in [K3].

\section{$3 \quad$ Wedge}

Lemma 3.1. (Dahlberg, see [D]) Let $u_{1}, u_{2}$ be two nonnegative harmonic functions in domain $D$ of the form

$$
D=\left\{(x, y) \in \mathbb{R}^{n} \times \mathbb{R}:|x|<1,|y|<M, y>f(x)\right\}
$$

with $f$ a Lipschitz function with constant less than $M$ and $f(0)=0$. Assume further that $u_{1}$ and $u_{2}$ take continuously the value $u_{1}=u_{2}=0$ along the graph of $f$. Then, on the domain

$$
\begin{gathered}
D_{1 / 2}=\{|x|<1 / 2,|y|<M / 2, y>f(x)\} \\
0<C_{1} \leq \frac{u_{1}(x, y)}{u_{2}(x, y)} \cdot \frac{u_{2}(0, M / 2)}{u_{1}(0, M / 2)} \leq C_{2}
\end{gathered}
$$

with $C_{1}, C_{2}$ depending only on $M$.

For a unit vector $\nu \in \mathbb{R}^{2}$ and $0 \leq \theta_{0} \leq 2 \pi$, define the sector, or wedge

$$
W\left(\theta_{0}, \nu\right):=\left\{z \in \mathbb{R}^{2}: z \cdot \nu \geq|z| \cos \frac{\theta_{0}}{2} \cdot\right\}
$$

We denote the vertical unit vector $(0,1)$ by $e_{2}$.

Dahlberg's estimate for a wedge in the plane is an elementary estimate, valid uniformly as the Lipschitz constant tends to infinity (and in the limiting case in which the wedge becomes the disk with a slit removed). This can be seen by observing that the conformal mapping $z=(x+i y) \rightarrow z^{k}, 1 \leq k \leq 2$ for appropriate $k$ depending on $\theta_{0}$, sends a wedge to the half disk, that is, the case $\theta_{0}=\pi$. We state this in the following lemma: 
Lemma 3.2. Let $u_{1}$ and $u_{2}$ be nonnegative harmonic functions in $W\left(\theta_{0},-e_{2}\right) \cap$ $B_{1}$ with $u_{i}=0$ on $\partial W\left(\theta_{0},-e_{2}\right) \cap B_{1}$ where $\pi \leq \theta_{0} \leq 2 \pi$. Normalize $u_{i}$ so that $u_{1}\left(-e_{2} / 2\right)=u_{2}\left(-e_{2} / 2\right)$. Then there exists $C_{1}, C_{2}>0$, independent of $\theta_{0}$ such that for $z \in W\left(\theta_{0},-e_{2}\right) \cap B_{1 / 2}$

$$
C_{1} \leq \frac{u_{1}(z)}{u_{2}(z)} \leq C_{2}
$$

These lemmas show that the order of magnitude of the initial harmonic function $u_{0}$ is determined by the shape of the boundary in the unit disk around the origin, and does not change by much if the boundary is modified outside that disk.

We will consider acute angles briefly before excluding them completely. We say that $u$ has a waiting time at $z \in \mathbb{R}^{2}$ if there exists $\epsilon>0$ such that $z \in \Gamma_{t}(u)$ for $0 \leq t \leq \epsilon$. It is proved in [KLV] using comparison with self-similar solutions that for any viscosity subsolution $u$ of (HS) with initial data $u_{0}, \Gamma(u)$ has a waiting time at a vertex only if the angle $\theta_{0}<\pi / 2$. We will now give a simple proof.

Proposition 3.3. Let $u$ be a viscosity subsolution of (HS) with initial data $u_{0}=H\left(1, \partial K, \Omega_{0}\right)$ with $u=1$ on $\partial K$ and $\Omega_{0}$ satisfying condition (A) with $\theta_{0}<\pi / 2$. Then the origin $(0,0)$ belongs to $\Gamma_{t}(u)$ for sufficiently small $t>0$. In other words, there is a waiting time before this free boundary point moves.

Proof. For $1<k \leq 2, z=(x, y)$, define

$$
\Phi(z, t)=\left(y^{2}-k x^{2}+25 t x^{2}\right)^{+} \text {if } y \leq 0, \text { otherwise zero. }
$$

Since $\Gamma(\Phi)=\{(z, t): y=-\sqrt{k-25 t}|x|\}$ we have

$$
\Phi_{t}-|\nabla \Phi|^{2}=25 x^{2}-4 y^{2}-(2 k-50 t)^{2} x^{2} \geq x^{2} \geq 0 \quad \text { on } \Gamma(\Phi)
$$

if $0 \leq t \leq 1 / 10$. Moreover

$$
-\Delta \Phi=-2+2 k-50 t>0 \quad \text { if } t \leq \frac{k-1}{25} .
$$

Thus $\Phi$ and $C \Phi(z, C t)$ are supersolutions of (HS) for $C t \leq \frac{k-1}{25}$.

There exist $C>0$ and $k, 1<k<2$, depending only on how close $\theta_{0}$ is to $\pi / 2$ such that $1 \leq C \Phi(z, 0)$ for $z \in\left(\partial B_{1}\right) \cap \Omega_{0}(\Phi)$. (Note that $k \rightarrow 1$ as $\left.\theta_{0} \rightarrow \pi / 2\right)$. By the maximum principle,

$$
u_{0}(z) \leq C \Phi(z, 0), \quad z \in B_{1} \cap \Omega_{0}(\Phi) .
$$

Next we claim that for small $t>0, u(z, t)=0$ for $z$ in a neighborhood of $\partial B_{1}-\Omega_{0}(\Phi)$. For this purpose we introduce a radial supersolution. For $a>0$, $b \geq 2 / a$, and $0 \leq t \leq a / 2 b$, define the function $\Psi(z, t)=(\log |z|-\log (a-b t))^{+}$ for all $z \in \mathbb{R}^{2}$. Then $\Psi$ (and hence $C \Psi(z, C t)$ ) is a supersolution. The free boundary is the circle $|z|=a-b t$, which shrinks from radius $a$ to radius $a / 2$. 
Since $u_{0}=0$ in a neighborhood of $\partial B_{1}-\Omega_{0}(\Phi)$ and $u \leq 1$, for a sufficiently small the maximum principle (Theorem 2.7 (a)) implies that a translate of the radial supersolution $C \Psi(z, C t)$, centered at any point of $\partial B_{1}-\Omega_{0}(\Phi)$, majorizes $u$ for small time $t$. This proves the claim.

The claim, combined with our earlier estimates, implies that for small $t$, $u^{*}(z, t)<C \Phi(z, C t)$ in $\partial B_{1}$. It follows from Theorem 2.7 (b) that $u(z, t) \leq C \Phi(z, C t)$ in $B_{1}$. In particular, $u(0, t)=0$ for small $t>0$.

From now on we will assume that in case A, $\theta_{0}>\pi / 2$. Note also that the cusps we will discuss later are very far from wedges with an acute angle, and more closely related to wedges with angle near $2 \pi$. We first prove the lower bound on the speed of the boundary (upper bound on $t(P)$ in (1.2)). This shows that the free boundary moves at the vertex. In particular, if $\Gamma_{0}$ can be represented locally as a Lipschitz graph with Lipschitz constant less than 1 , then Proposition 3.3 implies that $\Gamma_{0}$ moves immediately, so that Theorem 2.8 applies and says that there is a unique viscosity solution.

Proposition 3.4. Let $u$ be the minimal supersolution of (HS) with the initial data satisfying $(A)$ with $\pi / 2<\theta_{0}<2 \pi$ or $(B)$ or $(C)$. Then there is a absolute constant $C$ such that with the notations of Theorem 1.1,

$$
t(P) \leq C \delta^{2}(P) / u_{0}(\zeta(P))
$$

for $P \in B_{1}(0) \cap\left(\mathbb{R}^{2}-\bar{\Omega}\right)$.

Proof. For $P \in B_{1}(0) \cap\left(\mathbb{R}^{2}-\bar{\Omega}\right)$, let $\delta=\delta(P)$, then the ball of radius $\delta / 2$ around $\zeta(P)$ is in $\Omega$. For $|z| \geq 1$, consider

$$
\Psi(z, t)=\left(\log \left(\left(2+\frac{1}{25} t\right) /|z|\right) / \log \left(2+\frac{1}{25} t\right)\right)^{+}
$$

For $0 \leq t \leq 150, \Psi$ is a subsolution on $|z| \geq 1$. It follows that $C \Psi\left(r z, C r^{2} t\right)$ is a subsolution on $|z| \geq r$ with fixed boundary value $C>0$ on $|z|=r$ and free boundary equal to the circle $|z|=\left(2+\frac{1}{25} t\right) r$.

Harnack's inequality and the fact that $u$ increases in $t$ implies there is an absolute constant $c_{1}>0$ such that $u(z, t) \geq c_{1} u_{0}(\zeta(P))$ for $z \in B_{\delta / 4}(\zeta(P))$. Now we compare $u$ with

$$
h(z, t)=C \Psi\left(r(z-\zeta(P)), C r^{2} t\right)
$$

where $C=c_{1} u_{0}(\zeta(P))$ and $r=4 / \delta$. Then the free boundary at $t=0$ is $|z-\zeta(P)|=\delta / 2$, and the choice of $C$ implies that $h<u$ on $B_{\delta / 4}(\zeta(P))$. Thus $h \prec u$ at $t=0$. Hence by Theorem 2.7 (b), $h \leq u$. At the time $C r^{2} t=150$, $\Gamma_{t}(h)$ is the circle $|z-\zeta(P)|=2 \delta$, so that

$$
t(P) \leq 150 / C r^{2}=150 \delta^{2} / 16 c_{1} u_{0}(\zeta(P)) .
$$


Corollary 3.5. Suppose that $u$ is given as in Proposition 3.4. If (A) holds with $\pi / 2<\theta_{0}<2 \pi$, then there is a constant $c>0$ depending only on $\theta_{0}$ such that if $\alpha=2-\pi / \theta_{0}$

$$
\begin{cases}H_{1}(x, t) \geq c t^{1 / \alpha} & \text { if } t \geq|x|^{\alpha} ; \\ H_{1}(x, t) \geq c t|x|^{1-\alpha} & \text { if } t \leq|x|^{\alpha},\end{cases}
$$

or equivalently,

$$
H_{1}(x, t) \geq c \min \left[t^{1 / \alpha}, t|x|^{1-\alpha}\right] .
$$

Furthermore, if $3 \pi / 2 \leq \theta_{0}<2 \pi$, and $\beta=2 \pi-\theta_{0}$, then there is an absolute constant $c$ such that

$$
\begin{cases}H_{1}(x, t) \geq c(t / \beta)^{1 / \alpha} & \text { if } t / \beta \geq(|x| / \beta)^{\alpha} \\ H_{1}(x, t) \geq t|x|^{1-\alpha} \beta^{\alpha-2} & \text { if } t / \beta \leq(|x| / \beta)^{\alpha}\end{cases}
$$

or equivalently,

$$
H_{1}(x, t) \geq c \min \left[(t / \beta)^{1 / \alpha}, t|x|^{1-\alpha} \beta^{\alpha-2}\right] .
$$

Note that as $\theta_{0} \rightarrow 2 \pi, \beta \rightarrow 0$ and $\alpha \rightarrow 3 / 2$. To interpret geometrically the expressions $y_{1}(x, t)=t|x|^{1-\alpha} \beta^{\alpha-2}$ and $y_{0}(t)=(t / \beta)^{1 / \alpha}$ in the lower bound for $H_{1}$, for small $\beta>0$, observe that $y_{0} \leq y_{1}$ for $|x| / \beta \leq(t / \beta)^{1 / \alpha}$, and this range of $x$ is interpreted geometrically as $|x| \leq \beta y_{0}$. In other words, the set of $x$ for which the segment $\left(x, y_{0}\right)$ is in the complement of the wedge $\left(|x| \leq \beta y_{0}\right)$ the bound $H_{1} \geq c y_{0}$ is valid. For larger values of $x$, the bound is the smaller value $H_{1} \geq y_{1}$.

Proof. Suppose that $P=(x, y) \in \Gamma_{t}(u)$. Then $t=t(P)$ and $H_{1}(x, t) \simeq \delta(P)$. For the first pair of inequalities, we permit dependence on $\theta_{0}$ as it tends to $2 \pi$. We will keep track of the dependence on the distance $\beta$ from $2 \pi$ later. The size of $u_{0}$ is comparable to the explicit function positive homogeneous harmonic function of degree $2-\alpha$ with zero boundary values in the wedge. Thus,

$$
\begin{array}{ll}
u_{0}(\zeta(P)) \simeq \delta(P)|x|^{1-\alpha} & \text { for } \delta(P) \leq|x| ; \\
u_{0}(\zeta(P)) \simeq \delta(P)^{2-\alpha} & \text { for } \delta(P) \geq|x| .
\end{array}
$$

To interpret the bound $t(P) \leq \delta(P)^{2} / u_{0}(\zeta(P))$ as a bound on $H_{1}(x, t)$, consider first the case $\delta(P) \leq|x|$. Then by Proposition 3.4 (abbreviating $\delta=\delta(P)$ and $\left.u_{0}=u_{0}(\zeta(P))\right)$,

$$
\delta^{2} \geq c t u_{0} \geq c t \delta|x|^{1-\alpha} \Rightarrow H_{1} \simeq \delta \geq t|x|^{1-\alpha}
$$

Furthermore, combining this conclusion with $|x| \geq \delta$, one has $|x| \geq t|x|^{1-\alpha}$ and hence $t \leq C|x|^{\alpha}$. Next, consider the case $\delta \geq|x|$. Then by Proposition 3.4, $\delta^{2} \geq c t u_{0} \geq c t \delta^{2-\alpha}$, which implies $\delta^{\alpha} \geq c t$. Thus $H_{1} \simeq \delta \geq c t^{1 / \alpha}$, which is what 
we want to prove in the case $t \geq|x|^{\alpha}$. On the other hand, if $t<|x|^{\alpha}$, then, trivially, $\delta \geq|x| \geq t|x|^{1-\alpha}$.

Next we keep track of how these estimates depend on $\beta$ as $\theta_{0}$ tends to $2 \pi$. From now on the inequalities and $\simeq$ comparisons use absolute constants, not constants depending on $\beta$ as $\beta \rightarrow 0$. First, $H_{1} \simeq \delta / \beta$. Furthermore, $u_{0} \simeq \delta r^{1-\alpha}$, for $r=\sqrt{x^{2}+y^{2}}$. By Proposition 3.4,

$$
\delta^{2} \geq c t u_{0} \geq c t \delta r^{1-\alpha} \Rightarrow H_{1} \simeq \delta / \beta \geq c t r^{1-\alpha} / \beta
$$

Consider first the case $|x| \geq \delta$. Then since $|x| / \beta \geq \delta / \beta \simeq H_{1}, r \simeq|x| / \beta$. Thus,

$$
H_{1} \geq \operatorname{ct}(|x| / \beta)^{1-\alpha} / \beta
$$

in the case $|x| \geq \delta$.

Consider next, the case $|x| \leq \delta$. Then $r \simeq \delta / \beta$. Combining this with the estimate $\delta \geq c t r^{1-\alpha}$ of Proposition 3.4, one has $\delta^{\alpha} \geq c t \beta^{\alpha-1}$. Finally,

$$
H_{1} \simeq \delta / \beta \geq\left(c t \beta^{\alpha-1}\right)^{1 / \alpha} / \beta=(c t / \beta)^{1 / \alpha}
$$

Lemma 3.6. (Caffarelli, see [C2]) Let $u$ be given as in Lemma 3.1. There exist constants $C_{i}>0$ and $\delta>0$, depending only on $M$ (and dimension) such that

$$
C_{1} \frac{u(0, d)}{d} \leq \frac{\partial u}{\partial y}(0, d) \leq C_{2} \frac{u(0, d)}{d} \quad \text { for all } 0<d<\delta
$$

Lemma 3.7. (Caffarelli, see [C1]) Let $u$ be harmonic in $B_{1}$. Then there exists $\epsilon_{0}>0$ such that if $p$ is a unit vector and

$$
u(x+\epsilon p) \geq u(x) \text { for } \epsilon>\epsilon_{0} \text { and } x, x+\epsilon p \in B_{1},
$$

then $p \cdot D u \geq 0$ in $B_{1 / 2}$.

Proof of Theorem 1.2(a) with $\pi / 2<\theta_{0} \leq \pi$ :

1. The statement of Theorem $1.2(\mathrm{a})$ is equivalent to the claim

$$
H_{1}(x, t) \simeq \min \left[t^{1 / \alpha}, t|x|^{1-\alpha}\right] .
$$

Hence to prove the theorem it only remains to obtain the upper bound of $H_{1}(x, t)$. For this purpose we first construct a new free boundary which encloses $\Omega(u)$ outside of a local domain $\mathcal{O} \in \mathbb{R}^{2} \times[0, \infty)$ of the origin. Next, based on the new free boundary we construct a supersolution of (HS) in $\mathcal{O}$. Lastly we compare $u$ and our supersolution in $\mathcal{O}$ and apply the local maximum principle (Theorem 2.7(b)) to deduce the upper bound of $H_{1}(x, t)$.

2. Note that $0<\alpha \leq 1$. Let $0<r<1$. For each time $t \in\left[0, t_{0}\right], t_{0}=\frac{1}{4} r^{\alpha}$ we construct a free boundary $\Gamma(t)$ in $B_{2}(0) \cap\{|x| \leq 1\}$ via the following steps: (see figure 5). 


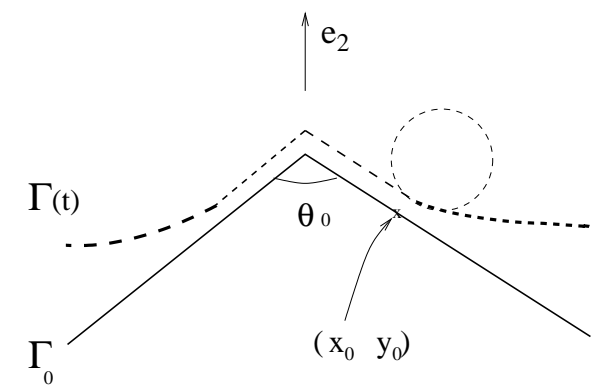

Figure 5.

(i) For $|x| \leq r$ : Let

$$
\Gamma_{1}(t)=\left[\Gamma_{0}+r^{1-\alpha} t e_{2}\right] \cap\{|x| \leq r\} .
$$

(ii) For $2 r \leq|x| \leq 1 / 4,(x, y(x, t)) \in \Gamma_{2}(t)$ if $\left(x, y_{0}(x)\right) \in \Gamma_{0}$ and

$$
y(x, t)=y_{0}(x)+|x|^{1-\alpha} t .
$$

(iii) For $r \leq|x| \leq 2 r$, we connect $\Gamma_{1}(t)$ and $\Gamma_{2}(t)$ with circular arcs (one for $x>0$ and one for $x<0$ ) so that the connected curve is $C^{1}$. We denote this arcs by $\tilde{\Gamma}_{1}(t)$. Note that the curvature of $\tilde{\Gamma}_{1}(t)$ is bounded by $r^{-1-\alpha} t \leq r^{-1}$.

(iv) For $1 / 4 \leq|x| \leq 1$ : we define $\Gamma_{3}(t)=\{(x, y(x, t)): 1 / 4 \leq|x| \leq 1\}$, where $\left(x, y_{0}(x)\right) \in \Gamma_{0}$ and

$$
y(x, t)=y_{0}(x)+\left(M_{0}(|x|-1 / 4)+1\right)|x|^{1-\alpha} t, \quad M_{0}>0
$$

Observe that for $z \in B_{2}(0)$,

$$
d\left(z, \Gamma_{0}\right) \geq \min \left[1, d\left(z, \Gamma_{0} \cap B_{2}(0)\right)\right] .
$$

Therefore by comparison with radial solutions one can easily check that for $0 \leq t \leq \delta$, there is $M>0$ such that

$$
\text { If }(x, y) \in \Gamma_{t}(u) \cap\{1 / 2 \leq|x| \leq 3 / 2\} \cap B_{2}(0) \text { then } H_{1}(x, t) \leq \frac{M}{4} t .
$$

Hence we can choose $M_{0}$ big enough in our construction of $\Gamma_{3}(t)$ such that if we let

$$
\Gamma_{4}(t)=\partial B_{1}(0) \cap\{y \geq y(1, t)\}
$$

then

$$
\Gamma(t)=\bigcup_{i=1,2,3,4} \Gamma_{i}(t) \cup \tilde{\Gamma}_{1}(t)
$$


is a closed curve enclosing the region $\Omega_{t}(u)-\{|x| \leq 1 / 2\} \cap B_{2}(0)$ for $0 \leq t \leq \delta$.

From now on we assume that $r^{\alpha} / 4 \leq \delta$ so that $t_{0} \leq \delta$. Let

$$
v(x, y ; t)=v^{t}(x, y)=H(1, \partial K, \Gamma(t)) .
$$

Based on $v$, we would like to construct a local supersolution of (HS) in the region $\{|x| \leq 1 / 2\} \cap B_{2}(0) \times\left[0, t_{0}\right]$ where $t_{0} \leq \delta$. For this purpose we estimate the normal velocity of the free boundary $\Gamma_{t}(v)$ and $|\nabla v|$ on $\Gamma(t)$ given as a limit from $\Omega_{t}(v)$. Observe that the normal velocity $V$ of $\Gamma(t)$ at $(x, y)$ (except at $x=0)$ satisfies that

$$
V \geq \begin{cases}\frac{1}{2} r^{1-\alpha} & \text { on } \Gamma_{1}(t) \cup \tilde{\Gamma}_{1}(t) \\ \frac{1}{2}|x|^{1-\alpha} & \text { on } \Gamma_{2}(t) \cup \Gamma_{3}(t)\end{cases}
$$

3. Thus it remains to estimate $|\nabla v|$ on $\Gamma(t)$. In the proof given below we denote $C_{i}$ 's as positive constants independent of $r$.

Estimates of $|\nabla v|$ on $\Gamma(t)$.

(i) Suppose $(x, y) \in \Gamma(t)$. If $1 / 8 \leq|x| \leq 3 / 4$, then $|\nabla v| \simeq|x|^{1-\alpha}$ by Harnack inequality and Lemma 3.1 .

(ii) Next we compare $v^{t}(x, y)$ with

$$
h(x, y)=H\left(1,-e_{2}, W\left(\theta+8 r^{\alpha}, e_{2}\right)+r e_{2}\right)
$$

for $|x| \leq 1 / 4$ and $t \leq t_{0}$. Since $v=0$ on $\Gamma_{t}(h)$ in $B_{1 / 4}(0)$ we obtain

$$
v^{t} \leq C_{1} h \text { on } B_{1 / 8}(0) \text {. }
$$

In particular $v^{t} \leq C_{1} h$ in $B_{t|x|}\left(x_{0}, y_{0}\right)$ for $\left(x_{0}, y_{0}\right) \in \Gamma_{2}(t) \cap\{|x| \leq 1 / 8\}$. Since at each point $\left(x_{0}, y_{0}\right) \in \Gamma_{2}(t)$ we have $\left(x_{0}, y_{0}+c r^{\alpha}\left|x_{0}\right|\right) \in \Gamma(h)$ with $1 / 2<c<2$,

$$
\bar{v}(x, t)=\bar{v}^{t}(x)=v^{t}\left(\left|x_{0}\right|\left(x-x_{0}\right),\left|x_{0}\right|\left(y-y_{0}\right)\right),
$$

satisfies $\bar{v}^{t}(0,-1 / 4) \leq C_{2}\left|x_{0}\right|^{2-\alpha-C_{3} r^{\alpha}}$. Since the curvature of $\Gamma_{2}(t)$ is bounded by $|x|^{-1-\alpha} t$, the curvature of $\Gamma_{t}(\bar{v})$ is bounded by $|x|^{-\alpha} t \leq 1 / 4$, and thus by comparison with radially symmetric harmonic functions and scaling back to $v$ we obtain

$$
\left|\nabla v^{t}\left(x_{0}, y_{0}\right)\right| \leq C_{4}\left|x_{0}\right|^{1-\alpha} \cdot r^{-C_{2} r^{\alpha}} \leq C_{5}\left|x_{0}\right|^{1-\alpha} \text { for } r / 2 \leq\left|x_{0}\right| \leq 1 / 8 .
$$

(iii) Finally for $|x| \leq r$ we observe that

$$
v^{t}(0,-r / 2) \leq C_{6} h(0,-r / 2) \leq C_{7} r^{2-\alpha-C_{3} r^{\alpha}} \leq C_{8} r^{2-\alpha},
$$

and hence again we apply Lemma 3.1 with $u_{2}=r^{2-\alpha} \cos (2-\alpha) \theta$ to obtain

$$
\left|\nabla v^{t}(x, y)\right| \leq C_{9}|x|^{1-\alpha} \text { for }|x| \leq r / 2 .
$$


4. From above estimates we obtain that $|\nabla v| \leq C|x|^{1-\alpha}$ on $\Gamma(t)$ for $|x| \leq 1$ where $C$ is independent of the choice of $r$. Now $\tilde{v}(x, y ; t)=v(x, y ; C t)$ satisfies

$$
\tilde{v}_{t} /|\nabla \tilde{v}| \geq C v_{t} /|\nabla v| \geq|\nabla \tilde{v}(x, y, t)| \text { on } \Gamma_{t} .
$$

Hence $\tilde{v}$ is a supersolution of (HS) in $\mathcal{O}:=\mathcal{N} \times\left[0, r^{\alpha} / 4\right]$ where $\mathcal{N}=\{|x|<3 / 4\} \cap B_{2}(0)$.

5. By definition $u^{*}=u=u_{0}$ at $t=0$ and thus

$$
u^{*}(z, t) \prec \tilde{v}_{\epsilon}:=\tilde{v}(z, t+\epsilon) \text { at } t=0
$$

for any small $\epsilon>0$. Suppose that $u^{*}-\tilde{v}_{\epsilon}$ has its first nonnegative maximum in $\overline{\Omega\left(u^{*}\right)}$ at $\left(x_{1}, y_{1}, t_{1}\right)$ with $t_{1} \leq t_{0}$. Since $u^{*}-\tilde{v}_{\epsilon}$ is subharmonic, $\left(x_{1}, y_{1}\right) \in \Gamma_{t}\left(u^{*}\right)$. Moreover by construction of $\Gamma(t),\left(x_{1}, y_{1}\right) \in \mathcal{N}$, which contradicts Theorem 2.7(b). Hence we obtain $u^{*} \prec \tilde{v}_{\epsilon}$ for $t \in\left[0,1 / 4 r_{0}^{\alpha}\right]$. Sending $\epsilon \rightarrow 0$ yields $\Gamma_{t}(u) \subset \overline{\Omega_{t}(\tilde{v})}$ and thus $u \leq \tilde{v}$ for $t \in\left[0, \frac{1}{4} r_{0}^{\alpha}\right]$. In particular

$$
H_{1}\left(x, \frac{1}{4 C} r^{\alpha}\right) \leq r \text { for }|x| \leq r, 0<r<1 .
$$

On the other hand

$$
H_{1}\left(x, \frac{1}{4 C} r^{\alpha}\right) \leq|x|^{1-\alpha} r^{\alpha} \text { for } r<|x|<1 / 4 .
$$

Since $0<r \leq(4 \delta)^{1 / \alpha}$ is arbitrary and $C$ does not depend on $r$, we conclude that

$$
H_{1}(x, t) \leq C \max \left(t^{1 / \alpha},|x|^{1-\alpha} t\right) \text { for } 0<|x|<1 / 4,0 \leq t \leq \delta .
$$

Now we can conclude from above inequality and Corollary 3.5.

Next we consider the rest of case A, that is when $\pi<\theta_{0}<2 \pi$.

Lemma 3.8. Let $u$ be given as in Theorem 1.2(a) with $\pi<\theta_{0}<2 \pi$. Then there is a constant $C=C\left(\theta_{0}\right)>0$ and $\delta$ : independent of $\theta_{0}$ such that

$$
H_{1}(x, t) \leq C \min \left[t^{1 / \alpha},|x|^{1-\alpha} t\right] \quad \text { for } 0 \leq|x|, t \leq \delta \tilde{\beta},
$$

where $\alpha=2-\pi / \theta_{0}$ and $\tilde{\beta}=\min \left(1, \tan \left[\pi-\theta_{0} / 2\right]\right)$.

Proof. 1. Below we only prove the lemma for $0<\beta<1$, that is when $3 \pi / 2<\theta_{0}<2 \pi$. For $\pi<\theta_{0} \leq 3 \pi / 2$, a parallel (and simpler) proof applies.

2. In the following proof positive constants $C_{i}$ 's only depend on $\theta_{0}$. Let us take $\left(r_{0} \beta, r_{0}\right) \in \Gamma_{0}$, for $0<r_{0}<1 / 4$. Then there is a ball $B_{r(0)}\left(\eta_{0}\right)$ of radius $r(0)=\beta \sqrt{\beta^{2}+1} r_{0}$ such that

$$
\bar{B}_{r(0)}\left(\eta_{0}\right) \cap \overline{\Omega\left(u_{0}\right)}=\left( \pm r_{0} \beta, r_{0}\right)
$$

(see figure 6.) We construct a free boundary $\Gamma(t)$ in $B_{2}(0) \cap\{y \leq 1\}$ as follows: For $0 \leq t \leq r_{0}^{\alpha} \beta$ we consider $r(t)=r(0)-\frac{1}{4} r_{0}^{1-\alpha} t$ and

$$
\Gamma_{1}(t)=\left\{(x, y) \in \Gamma_{0}+r(t) e_{2}:|x| \geq a(t)\right\}
$$




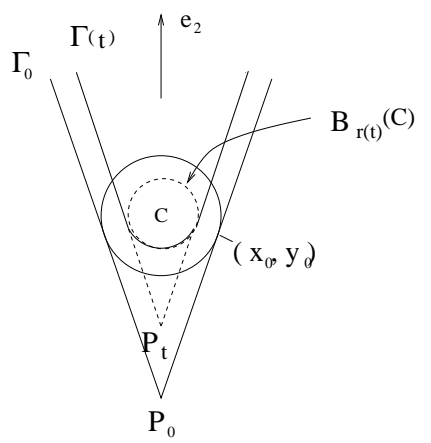

Figure 6.

where $a(t)$ is chosen such that $B_{r(t)}\left(\eta_{0}\right)$ is tangent to $\Gamma_{1}(t)$ at $|x|=a(t)$. Let $\Gamma_{2}(t)=\partial B_{r(t)}\left(\eta_{0}\right) \cap\{|x| \leq a(t)\}$ and consider

$$
\Gamma^{\prime}(t)=\Gamma_{1}(t) \cup \Gamma_{2}(t)
$$

(see figure 6.) Observe that the normal velocity $V$ of $\Gamma_{1}(t)$ satisfies

$$
V \geq \frac{1}{4} r_{0}^{1-\alpha} .
$$

3. Next we extend $\Gamma^{\prime}(t)$ for $1 \leq y$ as follows: first we let

$$
\Gamma_{3}(t)=\left\{(x, y):(x,-y+5 / 2) \in \Gamma^{\prime}(t), y \geq 3 / 2\right\}
$$

and we connect each end of $\Gamma^{\prime}(t)$ and $\Gamma_{3}(t)$ for $1 \leq y \leq 3 / 2$ by two smooth curves $\Gamma_{4}(t)$ whose curvature is bounded by 1 . Now we define

$$
\Gamma(t)=\cap_{i=1, . ., 4} \Gamma_{i}(t) .
$$

4. From now on we assume $r^{\alpha} \leq \delta$ so that $t \leq r^{\alpha} \beta \leq \delta \beta$. We consider $v(x, y ; t)=v^{t}(x, y)=H\left(1, \partial B_{4}(0), \Gamma(t)\right)$. First observe that, by the construction of $\Gamma(t),|\nabla v| \leq C_{1}$ on $\Gamma(t) \cap\{1 \leq y \leq 3 / 2\}$ by Lemma 3.1 and 3.2. By comparing $v^{t}$ with $\omega(x, y)=u_{0}\left(x, y-\frac{5}{4} r_{0}\right)$ and applying Lemma 3.1 at each point $\left(x_{0}, y_{0}\right) \in \Gamma_{1}(t)$ in $B_{4 r_{0} \beta_{0}}\left(x_{0}, y_{0}\right)$ it follows that

$$
\begin{gathered}
v^{t} \leq C_{2} r_{0}^{2-\alpha} \quad \text { in } B_{2 r_{0} \beta}\left(\frac{5}{4} r_{0} e_{2}\right) . \\
\left|\nabla v^{t}\right|(x, y) \leq C_{3}|x|^{1-\alpha} \quad \text { for }(x, y) \in \Gamma_{1}(t) .
\end{gathered}
$$

On the other hand since the curvature of $\Gamma_{2}(t)$ is bounded by $2\left(r_{0} \beta\right)^{-1}$, from (3.2) and comparison with radial solutions of (HS) in $B_{2 r_{0} \beta}\left(\frac{5}{4} r_{0} e_{2}\right)$ one obtains

$$
\left|\nabla v^{t}\right|(x, y) \leq C_{4} r_{0}^{1-\alpha} \text { for }(x, y) \in \Gamma_{2}(t) .
$$


5. From above estimates and by symmetry, there is $C_{5}>0$ such that $\tilde{v}(x, y ; t)=v\left(x, y ; C_{5} t\right)$ satisfies

$$
\tilde{v}_{t} /|\nabla \tilde{v}|-|\nabla \tilde{v}| \leq C_{5} r^{\prime}(t)-|\nabla v| \leq 0 \text { on } \Gamma(t) .
$$

Therefore $\tilde{v}$ is a supersolution of (HS) in $B_{4}(0) \times\left(0, C_{6} r_{0}^{\alpha} \beta\right)$. Now Theorem 2.7 (b) yields

$$
u \leq \tilde{v} \text { for } t \in\left[0, C_{6} r_{0}^{\alpha} \beta\right] .
$$

As a consequence it follows that,

$$
H_{1}\left(x, r_{0}^{\alpha} \beta\right) \leq C_{7} r_{0} \quad \text { for }|x| \leq r_{0} \beta .
$$

and

$$
H_{1}\left(r_{0} \beta, t\right) \leq C_{8}(r(t)-r(0))=\frac{C_{8}}{4} r_{0}^{1-\alpha} t \text { for } 0 \leq t \leq r_{0}^{\alpha} \beta .
$$

and we can conclude.

For the lower bound of $H_{1}(x, t)$, a straightforward computation using Lemma 3.6 and explicit values of $u_{0}$ leads to the following estimate:

Lemma 3.9. Let $u$ be as in Lemma 3.8 with $\pi<\theta_{0}<2 \pi$. Then there is a constant $C, \delta>0$ independent of $\theta_{0}$ that

$$
H_{1}(x, t) \geq C \min \left[\left(\frac{t}{\tilde{\beta}}\right)^{1 / \alpha}, \frac{|x|^{1-\alpha}}{\tilde{\beta}^{2-\alpha}} t\right] .
$$

for $0 \leq|x|, t \leq \delta \tilde{\beta}$, where $\tilde{\beta}=\min (1, \beta)$.

Proof of Theorem 1.2(a) with $\pi<\theta_{0}<2 \pi$ : $\quad$ It follows from Lemma 3.8 and Lemma 3.9.

Next we state and prove the $n$-dimensional version of Theorem 1.2(a). Let $\Omega$ be a bounded domain in $\mathbb{R}^{n}$ which satisfies

$$
\Omega \cap B_{3}(0)=\left\{x=\left(x^{\prime}, x_{n}\right) \in \mathbb{R}^{n}: x_{n} \leq\left|x^{\prime}\right| \cot \frac{\theta_{0}}{2}\right\} .
$$

We further suppose that $\Omega$ contains $K=B_{1}\left(-5 e_{n}\right), e_{n}=(0, . ., 1)$. We define $0<\theta_{n}<\pi$ be the dimensional constant such that with $\theta_{0}=\theta_{n}$ in (3.3) the harmonic function $u_{0}=H(1, K, \Omega)$ has quadratic decay at $x=0$, namely

$$
u_{0}\left(0,-r e_{n}\right)=O\left(r^{2}\right)
$$

for small enough $r$. We define $t(P), \delta(P)$ and $\zeta(P)$ as in (1.1)-(1.2). For a viscosity solution $u$ of (HS) with initial data $u_{0}$, we define $H_{1}\left(x^{\prime}, t\right)=x_{n}(x, t)-$ $x_{n}\left(x^{\prime}, 0\right)$ where

$$
x_{n}\left(x^{\prime}, t\right)=\sup \left\{x_{n}:\left(x^{\prime}, x_{n}\right) \in B_{1}(0) \cap \Gamma_{t}(u)\right\} .
$$

We also denote $a \simeq b$ if the $C_{1} a \leq b \leq C_{2} a$ for dimensional, positive constants $C_{1}$ and $C_{2}$. 
Theorem 3.10. Let $u(x, t)$ be a viscosity solution in $\mathbb{R}^{n} \times[0, \infty)$ with the initial data $u_{0}$ and fixed boundary data 1 and with $\theta_{n}<\theta_{0}<3 \pi / 2$. Then $u$ is unique and

$$
t(P) \simeq \delta(P)|P|^{\alpha-1}
$$

where $2-\alpha$ is the decay rate of $u_{0}$ at $x=0$, namely $u_{0}\left(0,-r e_{n}\right)=O\left(r^{2-\alpha}\right)$ for small $r$. In particular $\alpha \rightarrow 1$ as $\theta_{0}$ approaches $\pi$.

Since the proof of Theorem 3.10 is parallel to the case $n=2$, we only sketch the outline of the proof below.

Sketch of the proof for Theorem 3.10

1. As in the proof of Theorem 1.2(a), we will prove the equivalent statement in terms of $H_{1}\left(x^{\prime}, t\right)$ for $\left|x^{\prime}\right| \leq 1$ :

$$
H_{1}\left(x^{\prime}, t\right) \simeq \min \left[t^{1 / \alpha}, t|x|^{1-\alpha}\right] .
$$

First we need to prove the following lemma, which corresponds to Proposition 3.4.

Lemma 3.11. Let $u$ be the minimal supersolution of (HS) with $u_{0}, \theta_{0}$ and the fixed boundary data given as in Theorem 3.10. Then there is a dimensional constant $C$ such that

$$
t(P) \leq C \frac{\delta^{2}(P)}{u_{0}(\zeta(P))}
$$

for $P \in B_{1}(0) \cap\left(\mathbb{R}^{n}-\bar{\Omega}\right)$. In particular $t(P)=0$ if $P \in \Gamma_{0}(u)$ and $u$ is the unique viscosity solution of $(H S)$ with initial data $u_{0}$.

The last assertion of above lemma follows from Theorem 2.8. For the proof of the lemma we replace the barrier $\Psi$ in the proof of Proposition 3.4 to

$$
\Psi(x, t)=\frac{\left(\left(2+c_{n} t\right) /|x|\right)^{n-2}-1}{\left(2+c_{n} t\right)^{n-2}-1}
$$

where $c_{n}=5^{-n}$ and proceed similarly.

Corollary 3.12. Suppose that $u$ is given as in Lemma 3.11 and let $\alpha$ given as in Theorem 3.10. Then for $c>0$ depending only on $\theta_{0}$ and $n$,

$$
H_{1}\left(x^{\prime}, t\right) \geq c \min \left[t^{1 / \alpha}, t|x|^{1-\alpha}\right] .
$$

The proof of Corollary 3.12 is the same as that of Corollary 3.5.

2. It remains to obtain

$$
H_{1}\left(x^{\prime}, t\right) \leq C \min \left[t^{1 / \alpha}, t|x|^{1-\alpha}\right]
$$

for a dimensional constant $C$. As in the two-dimensional case we have to construct barrier functions separately for the cases $\theta_{n}<\theta_{0} \leq \pi$ and $\pi<\theta_{0}<3 \pi / 2$. For the first case we proceed as in the proof of Theorem 1.2(a). The main difference is in the construction of $\Gamma(t)$ in step 2.(a)-2.(d), defining $x_{n}(x, t)$ in 
different regions of the form $a<\left|x^{\prime}\right|<b$ instead of defining $y(x, t)$ in different regions of the form $a<|x|<b$. For the second case we proceed as in the proof of Lemma 3.8, where the main difference is again in the construction of $\Gamma^{\prime}(t)$ in terms of replacing the axis $e_{2}$ by $e_{n}$ and $(x, y)$ by $\left(x^{\prime}, x_{n}\right)$.

Now we proceed to prove Theorem 1.4. Here we use the self-similar solutions constructed in [KLV]. For $\pi / 2<\theta_{0}<2 \pi$ let us consider $U_{R}^{\theta_{0}}$ : a weak solution (see $[\mathrm{KLV}]$ for definition) of (HS) in $B_{R}(0) \times(0, \infty)$ with initial data $U_{R}(z, 0)=$ $r^{2-\alpha}[\cos (2-\alpha) \theta]_{+}$and fixed boundary data

$$
U_{R}^{\theta_{0}}(z, t)=R^{2-\alpha}[\cos (2-\alpha) \theta]_{+} \text {on } \partial B_{R}(0) .
$$

It was shown in $[\mathrm{KLV}]$ that $U_{R}^{\theta_{0}}(x, t)$ is increasing with respect to $t$ and $R$ and and locally uniformly converges to a continuous function $U^{\theta_{0}}$ in $\mathbb{R}^{n} \times[0, \infty)$ as $R \rightarrow \infty$, where $U^{\theta_{0}}(z, t)=t^{2 / \alpha-1} f\left(\frac{z}{t^{1 / \alpha}}\right)$ is a continuous self similar solution of (HS). In fact one can obtain exact formula for $U^{\theta_{0}}$ for $\pi / 2<\theta_{0}<2 \pi$ using conformal mappings (see Appendix in $[\mathrm{KLV}]$.)

\section{Proof of Theorem 1.4(a):}

1. For $R>1$ we consider

$$
u_{R}(z, t):=R^{2-\alpha} u\left(\frac{z}{R}, \frac{t}{R^{\alpha}}\right) \text { in } B_{R}(0) .
$$

We observe that there is a constant $A>0$ such that

$$
A:=\lim _{r \rightarrow 0} \frac{u_{0}(z)}{\left(r^{2-\alpha} \cos (2-\alpha) \theta\right)_{+}}
$$

Therefore we can choose $0<\delta=\delta(R)<A, \delta(R) \rightarrow 0$ as $R \rightarrow \infty$ which satisfies

$$
A-\delta \leq \frac{u_{R}(z, 0)}{\left[r^{2-\alpha} \cos (2-\alpha) \theta\right]_{+}} \leq A+\delta \text { in } \Omega_{0}\left(u_{R}\right) \cap B_{R}(0) .
$$

Note that $u_{R}$ is a viscosity solution of $(\mathrm{HS})$ in $B_{R}(0) \times(0,1)$.

2. First we consider the case $\pi / 2<\theta_{0}<\pi$. Due to Theorem 1.2(a) $d\left(\Gamma_{t}(u), \Gamma_{0}(u)\right) \leq C R^{-\alpha}$ for $0 \leq t \leq R^{-\alpha}$. Hence it follows that for any small $\epsilon>0$

$$
u_{R}^{*}(z, 1) \leq(A+\delta)(1+\epsilon) U_{R}^{\theta_{0}+\epsilon}(z, 0) \text { on } \partial B_{R}(0)
$$

if $R$ is sufficiently large. Since $U_{R}^{\theta_{0}} \leq U^{\theta_{0}}$ and $U^{\theta_{0}}$ is increasing in time, by Theorem 2.7

$$
u_{R}^{*}(z, t) \leq(A+\delta)(1+\epsilon) U^{\theta_{0}+\epsilon}(z,(A+\delta)(1+\epsilon) t) \text { in } B_{R}(0) \times[0,1]
$$

and

$$
\begin{aligned}
\lim \sup _{R \rightarrow \infty} u_{R}^{*}(z, t) & \leq \lim \sup _{\epsilon, \delta \rightarrow 0}(A+\delta) U^{\theta_{0}+\epsilon}(z,(A+\delta)(1+\epsilon) t) \\
& \rightarrow A U^{\theta_{0}}(z, A t)
\end{aligned}
$$


where the convergence follows from the explicit formula for $U^{\theta}$. In particular

$$
\limsup _{R \rightarrow \infty} u_{R}^{*}(z, 1) \leq A U^{\theta_{0}}(z, A t) \text { for }|z|, t \leq 1 .
$$

3. It is shown in [KLV] that for $\pi / 2<\theta_{0}<\pi$ there is $B>0,1 \leq \sigma<2-\alpha$ such that the following estimate holds:

$$
U^{\theta_{0}}(z, 1) \leq\left[R^{2-\alpha} \cos (2-\alpha) \theta+B R^{\sigma} \cos (\sigma \theta)+O(1)\right]_{+} \text {on } \partial B_{R}(0)
$$

It follows that for any small $\epsilon$ we have

$$
(A-\delta)(1-\epsilon) U^{\theta_{0}-\epsilon}(z,(A-\delta)(1-\epsilon) t) \leq u_{R}(z, t)
$$

if $R$ is sufficiently large. Hence one can argue as in step 1 to yield that

$$
A U^{\theta_{0}}(z, A t) \leq \liminf _{R \rightarrow \infty} u_{R}(z, t) \text { in }|z|, t \leq 1 .
$$

And thus $u_{R}$ uniformly converges to $A U^{\theta_{0}}(z, A t)$ in the domain $|z|, t \leq 1$. In particular, by self-similarity of $U^{\theta_{0}}$ there is $C=C\left(\theta_{0}\right)>0$ such that

$$
\lim _{R \rightarrow \infty} u_{R}(0, t)=C t^{1 / \alpha} \text {. }
$$

4. For $\pi<\theta_{0}<2 \pi$ similar arguments apply. In this case due to Theorem $1.2(\mathrm{~b})$

$$
d\left(\Gamma_{t}(u), \Gamma_{0}(u)\right) \leq C R^{-1} \text { for } 0 \leq t \leq R^{-\alpha}
$$

if $R$ is sufficiently large.

For the upper bound of the self-similar solution corresponding to (3.6), one can use a slight modification of the supersolution constructed in the proof of Lemma 3.8 to yield that

$$
U^{\theta_{0}}(z, 1) \leq\left[R^{2-\alpha} \cos (2-\alpha) \theta+O(1)\right]_{+} \text {on } \partial B_{R}(0) .
$$

The rest of the proof is parallel to that of step 2-3.

We next apply reflection arguments to show that the free boundary stays Lipschitz in space for small time.

Lemma 3.13. Let $u$ be a viscosity solution of (HS) with the initial data given in Case A. Then for any small constant $0<\tau<\min \left[\theta_{0}, 2 \pi-\theta_{0}\right]$, there exists $\delta, \rho>0$ depending on $\theta_{0}$ and $\tau$ such that $u$ is monotone increasing along $S:=$ $K\left(\theta_{0}-\tau,-e_{2}\right)$ in $B_{\rho} \times[0, \delta)$.

Proof. 1. Let us fix $0<\tau<\min \left[\theta_{0}, 2 \pi-\theta_{0}\right]$ and for each unit vector $p$ which has angle $\theta_{0}-k \tau$ with $-e_{2}$, consider

$$
l_{p}=\left\{z \in \mathbb{R}^{2}: z \cdot p=0\right\} .
$$

To prove the lemma we only have to show that $u$ is monotone increasing along $p \in \partial K$ in $B_{\rho} \times[0, \delta)$. Let $B_{1}^{+}$be the intersection of $B_{1}(0)$ and one side of $l_{p}$ in the direction of $p$. Depending on $\theta_{0}$ we choose $k<1$ such that $\Gamma_{0}$ is away from $l_{p}$ (see figure 7a-7c): then there are three cases which requires different analysis. 

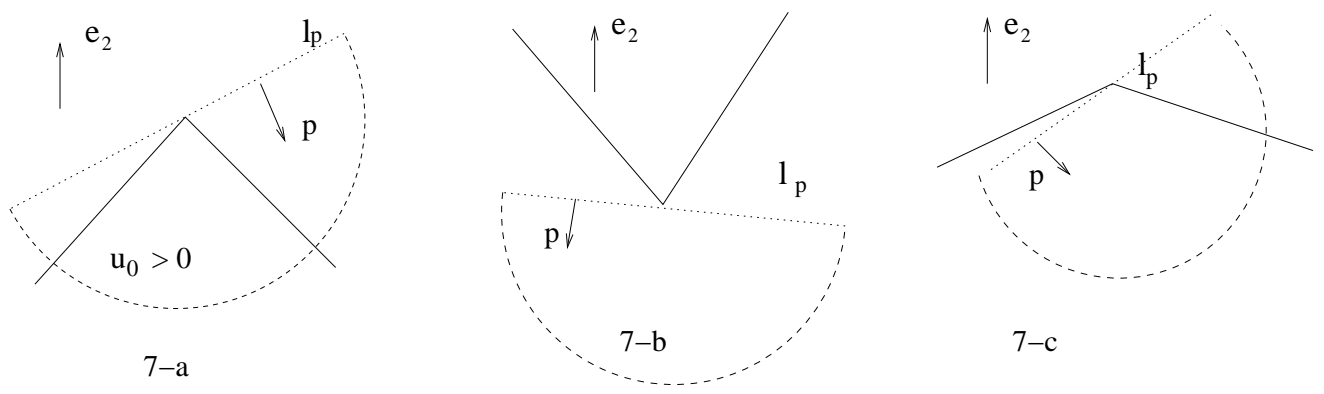

Figure 7.

(a) For $0<\theta_{0} \leq \pi / 2-\frac{3}{2} \tau$, we choose $k=1$ so that both legs of $\Gamma_{0}$ lies in $B_{1}^{+}$;

(b) For $\pi / 2+\frac{3}{2} \tau \leq \theta_{0}<2 \pi$, we choose $k=1$ so that both legs of $\Gamma_{0}$ lies in $B_{1}(0)-B_{1}^{+}$;

(c) For $\left|\theta_{0}-\theta_{0}\right| \leq \frac{3}{2} \tau$, we choose $k=3 / 4$ so that one leg of the wedge $\Gamma_{0} \cap B_{1}(0)$ lies in $B_{1}^{+}$and the other leg lies in $B_{1}-B_{1}^{+}$.

2. First let us assume (a). Set $\omega$ as the reflection of $u$ with respect to $l_{p}$. Observe that $\omega$ solves (HS) with $\omega=u$ on $l_{p}$. Let us choose $\delta=\delta(\tau)$ such that $\omega(z, t)=0$ on $\left(\partial B_{1}(0)-\partial B_{1}^{+}\right)$for $t \in[0, \delta)$. Since $\omega=0$ at $t=0$, by Theorem 2.7 we obtain

$$
(1-h) \omega^{*}(z, t-h) \leq u(z, t) \text { in } B_{1}^{+} \times[h, \delta)
$$

for any $h>0$. Hence by lowersemicontinuity of $\omega$ we obtain $\omega \leq u$ in $B_{1}^{+} \times[0, \delta)$. This implies that $u(z, t)$ is increasing in the direction of $p$ if $z \in l_{p} \cap B_{1}(0)$. The same argument follows for shifts of $l_{p}$ by small distance $\epsilon_{0}$ in the $\pm e_{2}$ direction with a smaller choice of $\delta>0$. (For shifts towards $-e_{2}$ direction, the argument works since $\omega \leq u$ in $B_{1}^{+}$at $t=0$ by comparison principle of harmonic functions. Hence $u$ is increasing along $K$ in the domain

$$
\Sigma=\cap_{p \in K} \cup\left\{l_{p}+\epsilon e_{2}:|\epsilon| \leq e_{0}\right\} \times[0, \delta),
$$

which contains a small cylinder $B_{\rho} \times[0, \delta)$.

3. Next we assume (b). In this case we apply Lemma 3.6 and 3.7. Due to Lemma 3.6, we may assume that $u_{0}(z)$ is monotone increasing along $S$ in $B_{1}$ and $u$ is indeed strictly increasing along $S$ in $\Omega(u) \cap B_{1}$. Moreover for any compact subset $D$ of $\Omega_{0}$, by the continuity of $u$ in time at $t=0$, for any $\epsilon>0$ there is $\delta>0$ such that $u$ is $\epsilon$-monotone along the direction $p$ in $D \times[0, \delta]$. Due to Lemma 3.4 if we choose $\epsilon$ small enough this yields to the full monotonicity of $u$ in the direction of $p$ in $D \times[0, \delta]$.

Now consider $\omega$ defined as in step 1 . We compare $u$ and $\omega$ in $B_{1}^{+}$. Let us choose a small neighborhood $\mathcal{N}$ of $\Gamma_{0}(\omega) \cap \partial B_{1}^{+}$so that $\omega(z, 0)<u_{0}(z)$ on $\mathcal{N}$ 


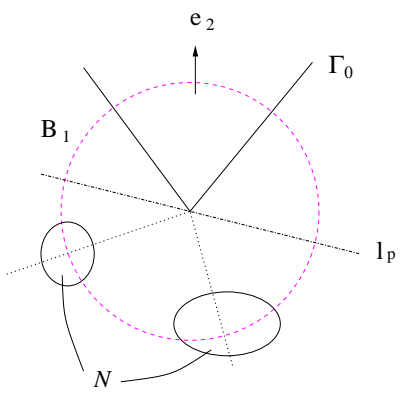

Figure 8 .

(see figure 8 above.) Due to above argument, there is $\delta>0$ such that

$$
\omega \leq u \text { on }\left(\partial B_{1}^{+}-\mathcal{N}\right) \times[0, \delta] .
$$

We choose $\delta$ small enough such that $\omega \leq u$ on $\partial B_{1}^{+} \times[0, \delta]$. Now $\omega \leq u$ on the parabolic boundary of $B_{1}^{+} \times[0, \delta]$ and the argument follows as in step 2 . to yield that $u$ is monotone increasing in the direction of $p$ in a small cylinder $B_{\rho}(0) \times[0, \delta]$.

4. Finally in the case of (c) combining above two arguments leads to the conclusion. Hence the lemma follows.

For the rest of this section we assume the additional hypotheses of Theorem 1.4(b): that is, we suppose that $\Omega_{0}$ is starshaped with respect to $P_{0}$ and $\Gamma_{0}$ is $C^{1,1}$ with respect to $P_{0}$. Next lemma provides a lower bound for the propagation speed of the free boundary for $\theta_{0}>\pi / 2$ :

Lemma 3.14. Let $u$ be as in Theorem 1.2(a) with $\pi / 2<\theta_{0}<2 \pi$. Then there exists $A, B>0$ depending on the size of the support of $u_{0}$ such that

$$
(1+A \epsilon) u\left((1+\epsilon) z,(1+A \epsilon)^{2} t+B \epsilon\right) \geq u^{*}(z, t) \text { for } t>0 .
$$

Proof 1. For $\pi \leq \theta_{0}<2 \pi$ the gradient $\left|\nabla u_{0}\right|$, formally the initial speed of propagation, is bounded from below on $\Gamma_{0}$ and hence it is easy to prove the lemma. The proof is parallel to Lemma 3.4 in [K3].

2. Hence we only consider cases $\pi / 2<\theta_{0}<\pi$. Due to Theorem 2.7 and the fact that $u_{t} \geq 0$, we only have to show that there is $A, B>0$ such that

$$
\omega(z, t)=(1+A \epsilon) u\left((1+\epsilon) z,(1+A \epsilon)^{2} t+B \epsilon\right)
$$

satisfies $\omega(z, 0) \geq u_{0}(z)$ for $z \in \partial K$. Since $\Gamma_{0}$ is preserved under dilation in $B_{1}(0)$, due to Theorem $1.2(\mathrm{a}) \omega(z, 0)>0$ on $\Gamma_{0} \cap B_{1}(0)$ for any $B>0$. Next we choose $B>0$ such that $\omega(\cdot, 0)>0$ on $\Gamma_{0}$ outside of $B_{1}(0)$. (Such $B$ exists since the support of $u_{0}$ is compact and outside of $B_{1}(0) \Gamma_{0}$ is $C^{1,1}$.) Finally since $|\nabla u|(\cdot, t)$ is bounded on $\partial K$ we can choose $A>0$ such that $\omega(\cdot, t) \geq 1$ on $\partial K$. Now the comparison principle for harmonic functions yields the conclusion. 
Remark Heuristically speaking, from Lemma 3.14 one obtains that

$$
\frac{u_{t}}{|\nabla u|}(z, t) \geq \frac{|z|}{2 A t+B} \text { for }(z, t) \in \Gamma(u) .
$$

\section{Proof of Theorem 1.4(b):}

Since $\Omega$ is starshaped with respect to the origin and since $u=1$ on $\partial K$ it follows that

$$
u(z, t) \geq u^{*}((1+\epsilon) z,(1+\epsilon) t) \text { in } Q
$$

Lemma 3.13 and above inequality with Lemma 3.14 provide the Lipschitz continuity of $u$ and $\Gamma(u)$ for small time $0 \leq t \leq \delta$. Moreover the nondegeneracy of $|\nabla u|$ on $\Gamma(u)$ follows from Lemma 3.14 (see [K3] for details.) Now the results in $[\mathrm{K} 2]$ yields the theorem.

\section{Cusp}

Note that Lemma 3.9 gives us a lower bound for the normal distance traveled by the free boundary with constants independent of $\theta_{0}$. We would like to obtain parallel result for the upper bound, so that we can apply these estimates to the cusp (B), that is when the opening of the cone $\theta_{0}$ tends to $2 \pi$ as we approach the vertex. Recall that for given $\pi / 2<\theta_{0}<2 \pi$, we define

$$
\beta_{0}=\pi-\theta_{0} / 2, \beta=\tan \beta_{0} \text { and } \alpha=2-\pi / \theta_{0} .
$$

In particular $\beta \rightarrow 0$ and $\alpha \rightarrow 3 / 2$ as $\theta_{0} \rightarrow 2 \pi$.

Lemma 4.1. Let $u$ be given as in Lemma 3.8 with $3 \pi / 2<\theta_{0}<2 \pi$. Then there are constants $\delta, C>0$ independent of $\theta_{0}$ such that for $0 \leq|x|, t \leq \delta \beta$ we have

$$
H_{1}(x, t) \leq C \min \left(\frac{t^{1 / \alpha}}{\beta^{1 / \alpha}}, \frac{|x|^{1-\alpha}}{\beta^{2-\alpha}} t\right) .
$$

Proof 1. Below we construct a supersolution of (HS) for $0 \leq t \leq \beta$ based on a free boundary $\Gamma(t)$ which we construct as follows. Note that $\alpha>1$.

(a) For $y_{t} \leq y \leq 1 / 2$ where $y_{t}=\left(\frac{t}{\beta}\right)^{1 / \alpha} \cdot \frac{1}{1-\beta}$, we define $\Gamma_{1}(t)$ as follows: $\left(x^{ \pm}(y, t), y\right) \in \Gamma_{1}(t)$ where

$$
x^{ \pm}(y, t)= \pm\left[\beta y-\frac{1}{4} \int_{0}^{t}\left(y-\left(\frac{s}{\beta}\right)^{1 / \alpha}\right)^{1-\alpha} d s\right] .
$$

(b) Next for $y \leq y_{t}$ we put a circular arc $\Gamma_{2}(t)$ connecting two points $\left(x^{ \pm}\left(y_{t}, t\right), y_{t}\right)$ so that the tangent line of $\Gamma_{2}(t)$ coincides with that of $\Gamma_{1}(t)$ at $y=y_{t}$. Let us consider $\Gamma(t)=\Gamma_{1}(t) \cup \Gamma_{2}(t)$. 
(c) For $y \geq 1 / 2$ we extend $\Gamma(t)$ as in the proof of Lemma 3.8 such that $\Gamma(t)$ has curvature less than 1 in the set $\{1 / 2 \leq y \leq 1\} \cap B_{2}(0)$ and $\Gamma(t)$ is symmetric with respect to axis $y=3 / 4$.

For simplicity let us assume $\delta=1$. We consider

$$
v(x, y ; t)=v^{t}(x, y)=H\left(1, \partial B_{4}(0), \Gamma(t)\right) .
$$

We would like to show that $v$ is a supersolution of (HS) for $0 \leq t \leq \beta$.

2. Normal velocity of $\Gamma(t)$.

(a) By definition the normal velocity of $\Gamma(t)$ at $y_{t}<y \leq 1$ is bigger than

$$
\frac{1}{2}\left|\partial_{t} x^{ \pm}(y, t)\right|=\frac{1}{8}\left(y-y_{t}+y_{t} \beta\right)^{1-\alpha} .
$$

(b) From a straightforward computation it follows that $\left|x^{ \pm}\left(y_{t}\right)\right|=c y_{t} \beta$ where $1 / 2<c<1$ is independent of $t$ and hence $\left|x^{ \pm}\left(y_{t}\right)\right|$ increases as $t$ increases. On the other hand one can verify that at $y=y_{t}$ the tangent line of $\Gamma(t)$ has slope $C \beta^{\alpha-1}$, where $C$ is independent of $t$. Therefore the curvature of $\Gamma_{2}(t)$ is decreasing with respect to $t$ and the normal velocity of $\Gamma_{2}(t)$ attains its minimum at $y=y_{t}$, which is

$$
\lim _{y \rightarrow y_{t}}\left[\text { the normal velocity of } \Gamma_{1}(t) \text { at }\left(x^{ \pm}(y, t), y\right)\right] \geq \frac{1}{4}\left(y_{t} \beta\right)^{1-\alpha} .
$$

Hence the normal velocity of $\Gamma_{2}(t)$ is greater than $\frac{1}{4}\left(y_{t} \beta\right)^{1-\alpha}$.

3. Estimate of $|\nabla v|$ on $\Gamma(t)$

(a) First observe that, since $\Gamma(t)$ has uniformly bounded curvature in $\Gamma(t) \cap$ $\{1 / 4 \leq y \leq 1\}$, by Harnack inequality and Lemma 3.1 we obtain $|\nabla v| \leq C_{0}$ in $\Gamma(t) \cap\{1 / 4 \leq y \leq 1\}$. Thus we only have to estimate $|\nabla v|$ on $\Gamma_{1}(t) \cup \Gamma_{2}(t)$.

(b) Below we denote $C$ as positive constants independent of $\beta$. For later use we compute the curvature $\kappa(y, t)$ of the free boundary $\Gamma_{1}(t)$ at $\left(x^{ \pm}, y\right), x^{ \pm}:=x^{ \pm}(y, t)$. Note that

$$
\left(y-y_{t}+y_{t} \beta\right) \leq\left(y-y_{s}+y_{s} \beta\right) \leq 2\left(y-y_{t}+y_{t} \beta\right) \text { for } t / 2 \leq s \leq t .
$$

Therefore

$$
\begin{aligned}
|\kappa(y, t)| & =\frac{\left|x_{y y}^{+}\right|}{\left(1+\left(x_{y}^{-}\right)^{2}\right)^{3 / 2}} \\
& \leq C\left(y-y_{t}+y_{t} \beta\right)^{-1-\alpha} t \cdot\left(1+\left(y-y_{t}+y_{t} \beta\right)^{-2 \alpha} t^{2}\right)^{-3 / 2} \\
\left.-y_{t}+y_{t} \beta\right)^{\alpha} & \leq t \text { then } \\
\kappa(y, t) & \leq C\left(y-y_{t}+y_{t} \beta\right)^{-1+2 \alpha} t^{-2} \leq C t^{-1 / \alpha} \leq C y_{t}^{-1} \beta^{-1 / \alpha} .
\end{aligned}
$$

If $\left(y-y_{t}+y_{t} \beta\right)^{\alpha} \leq t$ then

On the other hand if $\left(y-y_{t}+y_{t} \beta\right)^{\alpha} \geq t$ then

$$
\kappa(y, t) \leq C\left(y-y_{t}+y_{t} \beta\right)^{-1-\alpha} t \leq C t^{-1 / \alpha} \leq C y_{t}^{-1} \beta^{-1 / \alpha} .
$$


Hence we obtain

$$
|\kappa(y, t)| \leq C y_{t}^{-1} \beta^{-1 / \alpha} \leq C\left(y_{t} \beta\right)^{-1} .
$$

Also note that since $x^{ \pm}\left(y_{t}\right)=c y_{t} \beta$ with $1 / 2<c<1$,

$$
\Gamma_{2}(t) \text { has constant curvature } \kappa \leq 2\left(y_{t} \beta\right)^{-1} \text {. }
$$

For $0 \leq t \leq \beta$ let us consider

$$
h^{t}(x, y)=h(x, y ; t):=u_{0}\left(x, y-y_{t}\right) \text { in } B_{1 / 2} .
$$

Then $C_{0} \leq h^{t}(0,-1 / 4) \leq 1$ and $h^{t}>0$ in $\Omega_{t}(v) \cap B_{2}(0)$. Hence by Lemma 3.2 $v^{t} \leq C h^{t}$ in $B_{1 / 2}$. In particular

$$
v(\cdot, t) \leq C\left(y_{t} \beta\right)^{2-\alpha} \text { on } \partial B_{2 y_{t} \beta}\left(0, y_{t}\right) \text { for } 0 \leq t \leq \beta .
$$

(c) We scale

$$
\tilde{v}(x, y ; t)=\left(y_{t} \beta\right)^{\alpha-2} v\left(y_{t} \beta x,\left(y_{t} \beta\right)\left(y-y_{t}\right) ; t\right)
$$

so that $\tilde{v} \leq C_{3}$ on $\partial B_{1}$. Also from (4.1) and (4.2) the scaled free boundary $\Gamma_{t}(\tilde{v})$ has bounded curvature in $B_{1}(0)$. These facts and comparison with radially symmetric harmonic functions yields that

$$
|\nabla v(\cdot, t)| \leq C_{4}\left(y_{t} \beta\right)^{1-\alpha} \text { on } \Gamma_{2}(t) \text { for } 0 \leq t \leq \beta .
$$

(d) It remains to estimate the derivative on $\Gamma_{1}(t)$. Consider a ball of radius $y_{t} \beta$ centered in $\left(x_{0}, y_{0}\right) \in \Gamma_{1}(t)$ and $\tilde{v}(x, y ; t)=v\left(y_{t} \beta\left(x-x_{0}\right), y_{t} \beta\left(y-y_{0}\right) ; t\right)$. Again due to estimates (4.1)-(4.2) the curvature of $\Gamma_{t}(\tilde{v})$ is bounded independent of $\beta, t$. On the other hand $\Gamma_{t}(h)$ is at most $y_{t} \beta$ away from $\Gamma_{t}(v)$, and thus

$$
\tilde{v}(1 / 2,0 ; t) \leq C h^{t}\left(x_{0}+\frac{1}{2} y_{t} \beta, y_{0}\right) \leq C\left(y_{0}-y_{t}+y_{t} \beta\right)^{1-\alpha}\left(y_{t} \beta\right) .
$$

Hence by comparison with radially symmetric solutions and applying Lemma 3.1 , it follows that $\left|\nabla \tilde{v}\left(x_{0}, y_{0} ; t\right)\right| \leq C\left(y_{0}-y_{t}+y_{t} \beta\right)^{1-\alpha}$.

4. Above estimates show that $v_{t}-C|\nabla v|^{2} \geq 0$ for $C>0$ : independent of $\beta$ for $0 \leq t \leq \beta$. Hence $\bar{v}(x, y ; t):=v(x, y ; C t)$ is a supersolution of (HS) in $B_{4}(0) \times(0, \beta / C)$. Moreover $u_{0}(x, y) \leq \bar{v}(x, y ; 0)$ in $B_{4}(0)$, and hence due to Theorem 2.7

$$
u(x, y ; t) \leq \bar{v}(x, y ; t) \text { in } B_{4}(0) \times[0, \beta / C] .
$$

In particular it follows that for $0<r<1 / C$

(a) $H_{1}\left(x, r^{\alpha} \beta\right) \leq C r$ for $x \leq r \beta$;

(b) $H_{1}(r \beta, t) \leq C \frac{1}{\beta} r^{1-\alpha} t$ for $0 \leq t \leq r^{\alpha} \beta$, 


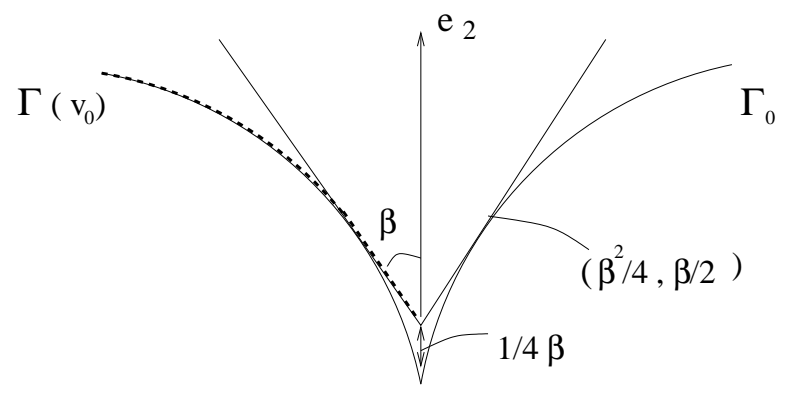

Figure 10.

which leads to the conclusion.

\section{Proof of Theorem 1.2(b):}

1. As before we will prove the equivalent statement

$$
H_{1}(x, t) \simeq \min \left[t^{2 / 5},|x|^{-3 / 4} t\right] .
$$

The lower bound of $H_{1}(x, t)$ follows from Proposition 3.4 and straightforward computation. Therefore it remains to obtain the upper bound of $H_{1}(x, t)$.

2 . For $\frac{3}{2} \pi \leq \theta_{0}<2 \pi$, Consider $v_{0}(x, y)$ : a harmonic function in $\Omega\left(v_{0}\right)$ with $v_{0}=1$ on $\partial K$ and

$$
\Gamma\left(v_{0}\right)= \begin{cases}\partial W\left(\theta_{0},-e_{2}\right)+\frac{1}{4} \beta e_{2} & \text { for }\left\{x:|x| \leq \beta^{2} / 4\right\} \cap B_{2}\left(P_{0}\right) \\ \Gamma_{0} & \text { otherwise }\end{cases}
$$

(see figure 10).

Since $\Omega\left(u_{0}\right) \subset \Omega\left(v_{0}\right), u_{0} \leq v_{0}$. Let $v$ solve (HS) with initial data $v_{0}$ and fixed boundary data $v=1$ on $\partial K$. Then $u \leq v$ and due to Lemma 4.1 there is $\delta>0$ such that

$$
H_{1}\left(x, \delta \beta^{\alpha+1}\right) \leq C \beta \text { for }|x| \leq \delta \beta^{2}
$$

and

$$
H_{1}\left(\delta \beta^{2}, t\right) \leq C \beta^{-\alpha} t \text { for } 0 \leq t \leq \delta \beta^{1+\alpha} .
$$

Since $0<\beta<1 / 2$ is arbitrary, we argue as in previous step to obtain

$$
H_{1}(x, t) \leq C \min \left(t^{2 / 5},|x|^{-3 / 4} t\right) \quad \text { for }|x|, t \leq \delta .
$$

\section{$5 \quad$ Touching fingers}

Lastly let us suppose that $\Omega_{0}$ is given as in $(\mathrm{C})$. Let us consider $u(x, y ; t)$ : a viscosity solution of (HS) with initial free boundary $\Gamma_{0}$ and fixed boundary data 


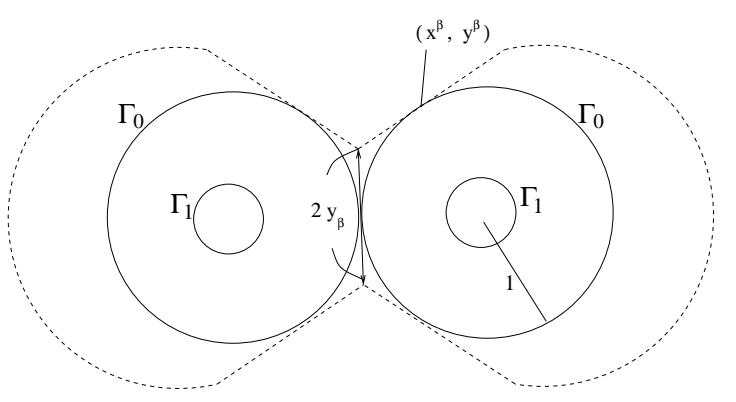

Figure 12 .

1 for $(x, y) \in \partial K$. Then $u_{0}=\sup \left(u_{0}^{1}, u_{0}^{2}\right)$, where

$$
u_{0}^{i}=H\left(1, \partial B_{1 / 10}\left((-1)^{i} e_{1}\right), \partial B_{1}\left((-1)^{i} e_{1}\right)\right) .
$$

Therefore

$$
u \geq \sup \left(u^{1}, u^{2}\right),
$$

where $u^{i}$ is the solution of (HS) with initial data $u_{0}^{i}$ and fixed boundary data 1 on $\partial B_{1 / 10}\left((-1)^{i} e_{1}\right)$. In particular, this implies that

$$
H_{1}(x, t) \geq C \min \left[t^{1 / 2},|x|^{-1 / 2} t\right] \text { for } 0 \leq|x|, t \leq 1 .
$$

On the other hand notice that for any $3 \pi / 2<\theta_{0}<2 \pi, u_{0} \leq v_{\theta_{0}}$, where $v_{\theta_{0}}=H\left(1, \partial K, \Gamma_{\theta_{0}}\right)$ with

$$
\Omega_{\theta_{0}}=\left[W\left(\theta_{0},-e_{2}\right)+y_{\beta} e_{2}\right] \cap\left[W\left(\theta_{0}, e_{2}\right)-y_{\beta} e_{2}\right]
$$

where

$$
y_{\beta}=\beta^{-1}\left[\sqrt{\beta^{2}+1}-1\right], \quad \frac{1}{2} \beta \leq y_{\beta} \leq \beta .
$$

Also one can easily verify that

$$
\Gamma_{\theta_{0}} \cap \Gamma_{0}=\left(x^{\beta}, y^{\beta}\right)=\left(\beta^{2}+1\right)^{-1 / 2}\left(\sqrt{\beta^{2}+1}-1, \beta\right) .
$$

(see figure 12.) For later use we state the following lemma:

Lemma 5.1. Let $v(x, y)$ be the harmonic function in $\mathbb{R}^{2}-\Gamma_{a}$ where

$$
\Gamma^{a}=\{(0, y): y \in \mathbb{R}-(0, a)\}
$$

with $v=0$ in $\Gamma^{a}$ and $v=1$ at $( \pm 1,0)$. Then $v(0, a / 2) \leq 2 a$.

Proof. Let $\Omega=\mathbb{R}^{2}-\Gamma^{a}$. Let us consider a conformal mapping $\omega=\frac{z}{z-a i}$. Then $\omega$ maps $\Omega$ one-to-one to $\mathbb{R}^{2}-\{(0, y): y \geq 0\}, \infty$ to $(0,1)$, and $( \pm 1,0)$ to $\left( \pm 1 /\left(1+a^{2}\right), a /\left(1+a^{2}\right)\right)$. Now we take $\zeta=\omega^{1 / 2}$ in $\Omega$ and observe that $\Omega$ is 
mapped one-to-one to $\mathbb{R}_{-}^{2}=\{(x, y): y<0\}, \zeta(\infty)=( \pm 1,0)$ and $\zeta(( \pm 1,0))=$ $P_{a}^{ \pm}$, where

$$
P_{a}^{ \pm}=\left( \pm\left(\frac{2+a^{2}}{2+2 a^{2}}\right)^{1 / 2}, \frac{a}{\left(2+2 a^{2}\right)^{1 / 2}}\right) .
$$

Let $\delta_{x}$ be the delta function at $(x, 0)$ and let $f$ be a harmonic function in $R_{-}^{2}$ such that $f=c\left[\delta_{1}+\delta_{-1}\right]$ on $\{y=0\}$ for some $c>0$ and $f=1$ at $P_{a}^{ \pm}$. Indeed if we choose $c$ small enough then

$$
f \leq 4 a\left[\frac{-y}{(x-1)^{2}+y^{2}}+\frac{-y}{(x+1)^{2}+y^{2}}\right]
$$

and hence $v=f(\zeta)$ satisfies our hypothesis with

$$
v(0, a / 2)=f(\sqrt{1 / 2},-\sqrt{1 / 2}) \leq 2 a .
$$

Corollary 5.2. Let $h(x, y)=H\left(1, \partial K, \Gamma_{2}\right)$ where $\Gamma_{2}=\partial\left[\Omega_{\theta_{0}} \cap B_{2}(0)\right]$. Then there is $c>0$ : independent of $\theta_{0}$ such that, for $(x, y) \in \Gamma_{\theta_{0}} \cap B_{1}(0)$,

$$
|\nabla h| \leq \begin{cases}c \beta^{\alpha-1}\left|y-y_{\beta}\right|^{1-\alpha} & \text { for } y-y_{\beta} \leq \beta / 2 \\ c & \text { for } \beta / 2 \leq y-y_{\beta} \leq 1 .\end{cases}
$$

Proof. 1. Below we denote $C$ as positive constants independent of $\theta_{0}$. Observe that by Lemma 5.1 and by comparison principle $h(0,0) \leq C \beta$. Hence the first inequality follows by Lemma 3.1 applied in $B_{\beta}(0,0)$.

2. Next for $(x, y) \in \Gamma_{\theta_{0}}$ with $|y| \geq \beta / 2$ ( we may assume that $x \geq 0$,) we compare $h$ with $v=\left(x-\beta\left(y-y_{\beta}\right)\right)_{+}$in $D:=B_{2}(0) \cap\left\{x \geq \beta^{2}\right\} \cap \Omega(v)$. Since $h \leq C v$ on $\partial B_{1 / 10}\left(e_{1}\right)$ and on $\partial D$ (due to the previous argument), it follows from Lemma 3.1 that $h \leq C v$ in $D$, which yields our assertion.

Now we are ready to prove that the inequality (5.1) holds in the other direction as well.

\section{Proof of Theorem 1.2(c):}

1. Let us choose $2 \pi-\theta_{0}$ small enough so that $0<\beta=\beta\left(\theta_{0}\right)<\delta$, where $\delta>0$ is to be chosen later. For given $\theta_{0}$, we change the coordinates $(x, y) \rightarrow\left(x, y-y_{\beta}\right)$ and construct a free boundary

$\Gamma^{+}(t)=\bigcup_{i=1,2,3} \Gamma_{i}(t)$ for $0 \leq t \leq \beta^{2}$ for $0<y \leq 1 / 2$ as follows.

(a) $\Gamma_{1}(t)=\left\{\left(x^{ \pm}(y, t), y\right): 2 y_{t} \leq y \leq 1 / 2\right\}$ where $y_{t}=t^{1 / 2}(1-\beta)^{-1}$ and

$$
x^{ \pm}(y, t)= \pm(\beta y-t) .
$$

(b) $\Gamma_{2}(t)=\left\{\left(x^{ \pm}(y, t), y\right): y_{t} \leq y \leq 2 y_{t}\right\}$ where

$$
x^{ \pm}(y, t)= \pm\left[\beta y-(1-\beta)^{2} y^{2} / 4-\int_{(1-\beta)^{2} y^{2} / 4}^{t}(\beta / 2)^{\alpha-1}\left(y-s^{1 / 2}\right)^{1-\alpha} d s\right] .
$$

Observe that $\Gamma^{+}(t)$ is $C^{1}$ at $y=2 y_{t}$. 
(c) For $y \leq y_{t}$ we construct $\Gamma_{3}(t)$ as a circular arc connecting two endpoints of $\Gamma_{2}(t)$ at $y=y_{t}$ so that $\Gamma^{+}(t)$ is $C^{1}$ at these points.

We define

$$
\Gamma(t)=\Gamma^{+}(t) \cup \Gamma^{-}(t), \Gamma^{-}(t)=\left\{(x, y):\left(x,-y-2 y_{\beta}\right) \in \Gamma^{+}(t)\right\} .
$$

Lastly we connect $\Gamma(t)$ and $\partial\left[B_{2}\left(e_{1}\right) \cup B_{2}\left(-e_{1}\right)\right] \cap\{|y| \geq 3 / 4\}$ with straight lines. By barrier arguments with radial solutions one can easily check that the region $\Omega(t)$ with boundary $\partial K$ and $\Gamma(t)$ includes $\Omega_{t}(u)-\{|y| \leq 1 / 2\}$ for $0 \leq t \leq \beta^{2}$. Consider

$$
v(x, y ; t)=v^{t}(x, y)=H(1, \partial K, \Gamma(t)) .
$$

We would like to show that $v$ is a supersolution of (HS) in $\{|y| \leq 1 / 2\} \times\left[0, \beta^{2}\right]$. In particular in the series of computations below we always assume $0 \leq t \leq \beta^{2}$.

2. Normal velocity of $\Gamma(t)$.

The normal velocity $V$ of $\Gamma_{i}(t)$ at $\left(x^{ \pm}(y, t), y\right), i=1,2$ is greater than $\frac{1}{2}\left|\partial_{t}\left(x^{ \pm}\right)(y, t)\right|$, where

$$
\left|\partial_{t}\left(x^{ \pm}\right)(y, t)\right| \geq \begin{cases}1 & \text { if }\left(x^{ \pm}, y\right) \in \Gamma_{1}(t), \\ (\beta / 2)^{\alpha-1}\left(y-y_{t}+y_{t} \beta\right)^{1-\alpha} & \text { if }\left(x^{ \pm}, y\right) \in \Gamma_{2}(t) .\end{cases}
$$

Next to estimate the normal velocity of $\Gamma_{3}(t)$, we compute $x^{+}\left(y_{t}, t\right)$ :

$$
\begin{array}{ll}
x^{+}\left(y_{t}, t\right) & =\beta y_{t}-\frac{(1-\beta)^{2}}{4} y_{t}^{2}-\int_{t / 4}^{t}(\beta / 2)^{\alpha-1}\left(y_{t}-s^{1 / 2}\right)^{1-\alpha} d s \\
\left((1-\beta) y_{t}=t^{1 / 2}\right) & =\beta y_{t}-\frac{(1-\beta)^{2}}{4} y_{t}^{2}-c \beta^{\alpha-1} y_{t}^{3-\alpha}
\end{array}
$$

where $1 / 4<c<1 / 2$ is independent of $t$. Since $1<\alpha<\frac{3}{2}$ and $y_{t} \leq \beta /(1-\beta)$ we obtain

$$
\frac{1}{8} \beta y_{t} \leq x^{+}\left(y_{t}, t\right) \leq \beta y_{t}
$$

for $0<\beta<1 / 10$. Moreover $x^{+}\left(y_{t}, t\right)$ increases as $t$ increases. Furthermore straightforward computation as above yields that $\partial_{t}\left(x^{+}\right)_{y}\left(y_{t}, t\right)>0$ if $\beta$ is sufficiently small. In particular the tangential slope of $\Gamma_{2}(t)$ at $y=y_{t},\left(x_{y}^{+}\right)^{-1}\left(y_{t}, t\right)$, decreases as time increases. Since $x^{+}\left(y_{t}, t\right)$ is increasing with respect to $t$, this implies that the curvature of $\Gamma_{3}(t)$ is decreasing with respect to time. Hence it follows that the normal velocity of $\Gamma_{3}(t)$ attains its minimum at $y=y_{t}$, which is

$$
\lim _{y \rightarrow y_{t}}\left[\text { the normal velocity of } \Gamma_{2}(t) \text { at }\left(x^{+}(y, t), y\right)\right] \geq \frac{1}{2} y_{t}^{1-\alpha} .
$$

Hence it follows that

$$
\text { the normal velocity of } \Gamma_{3}(t) \geq \frac{1}{2} y_{t}^{1-\alpha} \text {. }
$$




\section{Estimate of $|\nabla v|$ on $\Gamma(t)$.}

(i) As before we compute the curvature $\kappa(y, t)$ of the free boundary $\Gamma(t)$ at $\left(x^{+}(y, t), y\right), 0<y \leq 1 / 2$. First observe that $\Gamma_{I}(t)$ has curvature zero. Next observe that from above estimates we have $\left|\left(x^{+}\right)_{y}(y, t)\right| \leq \beta^{2-\alpha} \leq 1$. Hence on $\Gamma_{2}(t)$ we have

$(5.4)$

$$
\begin{aligned}
|\kappa(y, t)| \leq & 2\left|\left(x^{+}\right)_{y y}(y, t)\right| \\
\leq & (\alpha-1)^{2} \int_{(1-\beta)^{2} y^{2} / 4}^{t}(\beta / 2)^{\alpha-1}\left(y-s^{1 / 2}\right)^{-\alpha-1} d s \\
& +4 \beta^{\alpha-1} y^{1-\alpha} \\
& \\
\left(t \leq(1-\beta)^{2} y^{2}\right) \leq & \int_{(1-\beta)^{2} y^{2} / 4}^{(1-\beta)^{2} y^{2}}(\beta / 2)^{\alpha-1}\left(y-s^{1 / 2}\right)^{-\alpha-1} d s+4 \beta^{\alpha-1} y^{1-\alpha} \\
\left(s=(1-\beta)^{2} y^{2} \gamma\right) \leq & y^{1-\alpha} \beta^{\alpha-1} \int_{1 / 4}^{1}\left(1-(1-\beta) \gamma^{1 / 2}\right)^{-\alpha-1} d \gamma+4 \beta^{\alpha-1} y^{1-\alpha} \\
\leq & y^{1-\alpha} \beta^{-1}+4 \beta^{\alpha-1} y^{1-\alpha} \\
\leq & 5 y_{t}^{-1} \beta^{-1}
\end{aligned}
$$

Moreover note that, since $\left|x^{ \pm}\left(y_{t}, t\right)\right|>y_{t} \beta / 8$,

$$
\Gamma_{3}(t) \text { has constant curvature } \kappa \leq 8\left(y_{t} \beta\right)^{-1} \text {. }
$$

As before we denote $C$ as positive constants independent of $\beta$. Next we consider $h^{t}=H\left(1, \partial K, \Gamma_{t}(h)\right)$ where

$$
\Gamma_{t}(h)=\partial\left[\Omega_{t} \cap B_{2}\right]
$$

and

$$
\Omega_{t}=\left[W\left(\theta_{0},-e_{2}\right)+y_{t} e_{2}\right] \cap\left[W\left(\theta_{0}, e_{2}\right)-\left(y_{t}+2 y_{\beta}\right) e_{2}\right]
$$

Due to Corollary 5.2 there exists $C_{1}>0$ such that

$$
\left|\nabla h^{t}\right| \leq \begin{cases}C\left(y_{t}\right)^{\alpha-1}\left|y-y_{t}\right|^{2-\alpha} & \text { if }\left|y-y_{t}\right| \leq y_{\beta} \\ C & \text { if }\left|y-y_{t}\right| \geq y_{\beta}\end{cases}
$$

Note that $d\left(z, \Gamma_{t}(h)\right) \leq y_{t} \beta$ for $z \in \Gamma(t) \cap\{|y| \leq 1 / 2\}$. Now due to estimates (5.4) and (5.5) one can proceed as in the proof of Lemma 4.1 to compare $v$ and $h^{t}$ and to deduce that there exists $C_{2}>0$ such that

$$
|\nabla v(\cdot ; t)| \leq\left\{\begin{array}{l}
C \text { on } \Gamma_{1}(t) \\
C(\beta / 2)^{\alpha-1}\left|y-y_{t}+y_{t} \beta\right|^{1-\alpha} \text { on } \Gamma_{2}(t) \\
C \beta^{1-\alpha} \text { on } \Gamma_{3}(t) .
\end{array}\right.
$$


Thus $\tilde{v}(x, y ; t)=v(x, y ; C t)$ is a supersolution of (HS) in $\{|y| \leq 1 / 2\} \times\left[0, \beta^{2} / C_{2}\right]$. Since $u_{0} \leq \tilde{v}(x, y ; 0)$, proceeding as in the proof of Theorem 1.2(a) it follows that $u \leq \tilde{v}$ for $0<t<\beta^{2} / C$. Now we obtain

$$
H_{1}\left(x, \beta^{2} / C_{2}\right) \leq C \beta \text { for }|x| \leq \beta^{2} .
$$

Moreover, since $\left(x^{\beta}, y^{\beta}\right) \in \Gamma_{0}$ (see (5.3)) and $y^{\beta} \geq 2 y_{t}$ for $0 \leq t \leq \beta^{2} / 4$, we can choose $C$ large enough that

$$
\left.H_{1}\left(x^{\beta}, t\right) \leq \text { the normal velocity of } \Gamma_{1}(t)\right] \cdot \frac{t}{\beta} \leq C t / \beta \text { for } 0 \leq t \leq \beta^{2} / C .
$$

Since $0<\beta<\delta$ is random with sufficiently small $\delta$ and $\beta^{2} / 4 \leq x^{\beta} \leq \beta^{2}$, we obtain that

$$
H_{1}(x, t) \leq C \min \left(t^{1 / 2},|x|^{-1 / 2} t\right) \text { for } 0 \leq|x|, t \leq \delta
$$

and thus we can conclude due to above estimate and (5.1).

\section{References}

[ACS] I. Athanasopoulos, L. Caffarelli and S. Salsa, Regularity of the free boundary in parabolic phase-transition problems, Acta Math., 176(1996), 245-282.

[C1] L. Caffarelli, A Harnack inequality approach to the regularity of free boundaries, Part II: Flat free boundaries are Lipschitz,Comm. Pure Appl. Math., 42(1989), 55-78.

[C2] L. Caffarelli, A Harnack inequality approach to the regularity of free boundaries, Part I: Lipschitz free boundaries are $C^{1, \alpha}$, Rev. Mat. Iberoamericana 3 (1987), no. 2, 139-162

[D] B.E. Dahlberg, Harmonic functions in Lipschitz domains, Harmonic analysis in Euclidean spaces, Part 1, pp. 313-322, Proc. Sympos. Pure Math., XXXV, Part, Amer. Math. Soc., Providence, R.I., 1979

$[\mathrm{H}] \quad$ S.D. Howison, Cusp development in Hele-Shaw flow with a free surface,SIAM J. Appl. Math.46 (1986), 20-26.

[K1] I. Kim, Uniqueness and Existence result of Hele-Shaw and Stefan problem, Arch. Rat. Mech. Anal., published online.

[K2] I. Kim, Regularity of free boundary in one phase Hele-Shaw problem, preprint.

[K3] I. Kim, Long time regularity of Hele-Shaw and Stefan problem, preprint.

[KLV] J.R. King, A.A. Lacey and J.L. Vazquez, Persistence of corners in free boundaries in Hele-Shaw flow, Euro. J. Appl. Math., 6(1995), 455-490. 